\title{
Uniformity, Universality, and Computability Theory
}

\author{
Andrew S. Marks* \\ September 1, 2018
}

\begin{abstract}
We prove a number of results motivated by global questions of uniformity in computability theory, and universality of countable Borel equivalence relations. Our main technical tool is a game for constructing functions on free products of countable groups.

We begin by investigating the notion of uniform universality, first proposed by Montalbán, Reimann and Slaman. This notion is a strengthened form of a countable Borel equivalence relation being universal, which we conjecture is equivalent to the usual notion. With this additional uniformity hypothesis, we can answer many questions concerning how countable groups, probability measures, the subset relation, and increasing unions interact with universality. For many natural classes of countable Borel equivalence relations, we can also classify exactly which are uniformly universal.

We also show the existence of refinements of Martin's ultrafilter on Turing invariant Borel sets to the invariant Borel sets of equivalence relations that are much finer than Turing equivalence. For example, we construct such an ultrafilter for the orbit equivalence relation of the shift action of the free group on countably many generators. These ultrafilters imply a number of structural properties for these equivalence relations.
\end{abstract}

${ }^{*}$ The author was partially supported by the National Science Foundation under grants DMS-1204907 and DMS-1500974 and the Turing Centenary research project "Mind, Mechanism and Mathematics", funded by the John Templeton Foundation under Award No. 15619 . 


\section{Contents}

1 Introduction 3

1.1 Introduction . . . . . . . . . . . . . . . . 3

1.2 Basic definitions, notation, and conventions . . . . . . . 11

1.3 Acknowledgments . . . . . . . . . . . . . . . 13

2 Games and equivariant functions 14

2.1 The main game . . . . . . . . . . . . . . . . . 14

2.2 The free part of the shift action . . . . . . . . . 18

2.3 Results in reverse mathematics ............ 21

3 Uniform universality 24

3.1 Introduction to uniform universality . . . . . . . . . . . 24

3.2 Basic results on uniform universality . . . . . . . . . . 27

3.3 Limitations on controlling countable joins . . . . . . . . . . 30

3.4 Uniform universality and equivalence relations from computabil-

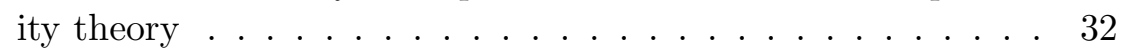

3.5 Contrasting results in the measure context and on $3^{\omega} \ldots . .37$

4 Ultrafilters on quotient spaces

4.1 An introduction to ultrafilters on quotient spaces . . . . . . 45

4.2 A natural ultrafilter on the quotient of $E\left(\mathbb{F}_{\infty}, 2^{\omega}\right) \ldots \ldots$

$4.3 \mathcal{K}$-structurable equivalence relations . . . . . . . . . . 54

4.4 Ultrafilters for $\mathcal{K}$-structurable equivalence relations closed under independent joins . . . . . . . . . . . . 56 


\section{Introduction}

\section{$1.1 \quad$ Introduction}

In this paper, we investigate a number of problems concerning uniformity and universality among countable Borel equivalence relations and in computability theory.

In a sense, this paper is a sequel to [28]. In that paper, we used Borel determinacy to prove a Ramsey-like theorem ([28, Lemma 2.1]) for the shift action of a free product of two groups. We then gave applications of this result to several problems in Borel graph combinatorics. In this paper, we generalize the games used in 28] to handle shift actions of free products of countably many groups. The resulting theorems we prove above these shift actions are the main technical tool of this paper. However, the applications we give in this paper are to problems in the study of countable Borel equivalence relations and computability theory, rather than Borel graph combinatorics.

We briefly mention two of the results proved using these games. Recall that if a group $\Gamma$ acts on the spaces $X$ and $Y$ then a function $f: X \rightarrow Y$ is said be $\Gamma$-equivariant if $f$ commutes with the actions of $\Gamma$ on $X$ and $Y$, so $\gamma \cdot f(x)=f(\gamma \cdot x)$ for every $x \in X$ and $\gamma \in \Gamma$. If $\Gamma$ is a countable discrete group and $X$ is a Polish space, then $\Gamma$ acts on the Polish space $X^{\Gamma}$ via the left shift action where for all $x \in X^{\Gamma}$ and $\gamma, \delta \in \Gamma$

$$
\gamma \cdot x(\delta)=x\left(\gamma^{-1} \delta\right)
$$

One importance of the shift action is that it is universal in the sense that every Borel action of $\Gamma$ on a Polish space $X$ admits a Borel embedding into the shift action by sending $x \in X$ to the function $\gamma \mapsto \gamma^{-1} \cdot x$. We will use the notation $*_{i} \Gamma_{i}$ to denote the free product of the groups $\left\{\Gamma_{i}\right\}_{i \in I}$.

Theorem 1.1. Suppose $I \leq \omega$ and $\left\{\Gamma_{i}\right\}_{i \in I}$ is a set of countable discrete groups. Let $\left\{A_{i}\right\}_{i \in I}$ be a Borel partition of $\left(2^{\omega}\right)^{*_{i} \Gamma_{i}}$. Then there exists some $j \in I$ and an injective continuous function $f:\left(2^{\omega}\right)^{\Gamma_{j}} \rightarrow\left(2^{\omega}\right)^{*_{i} \Gamma_{i}}$ that is $\Gamma_{j}$ equivariant with respect to the shift actions, and such that $\operatorname{ran}(f) \subseteq A_{j}$. Furthermore, $f$ can be chosen to be a Borel reduction between the orbit equivalence relations induced by the shift actions of $\Gamma_{j}$ on $\left(2^{\omega}\right)^{\Gamma_{j}}$ and $*_{i} \Gamma_{i}$ on $\left(2^{\omega}\right)^{*_{i} \Gamma_{i}}$.

That is, if we partition the shift action of the group $*_{i \in I} \Gamma_{i}$ into $I$ many Borel sets $\left\{A_{i}\right\}_{i \in I}$, not only does one of the sets $A_{j}$ contain a copy of the 
shift action of $\Gamma_{j}$ (as witnessed by a continuous equivariant injection), the Borel cardinality of the shift action of $\Gamma_{j}$ is also preserved.

We also have a version of this theorem for the free part of the action. Given a group $\Gamma$ and a Polish space $X$, let

$$
\operatorname{Free}\left(X^{\Gamma}\right)=\left\{x \in X^{\Gamma}: \operatorname{Stab}(x)=\{1\}\right\}
$$

be the free part of the left shift action: the largest set where every stabilizer under this action is trivial. We have an analogous theorem for the free parts of these actions:

Theorem 1.2. Suppose $I \leq \omega$ and $\left\{\Gamma_{i}\right\}_{i \in I}$ is a set of countable discrete groups. Let $\left\{A_{i}\right\}_{i \in \omega}$ be a Borel partition of Free $\left(\left(2^{\omega}\right)^{*_{i} \Gamma_{i}}\right)$. Then there exists some $j \in I$ and an injective continuous function $f:$ Free $\left(\left(2^{\omega}\right)^{\Gamma_{j}}\right) \rightarrow$ Free $\left(\left(2^{\omega}\right)^{*_{i} \Gamma_{i}}\right)$ that is $\Gamma_{j}$-equivariant with respect to the shift actions, and such that $\operatorname{ran}(f) \subseteq A_{j}$. Furthermore, $f$ can be chosen to be a Borel reduction between the orbit equivalence relations induced by the shift actions of $\Gamma_{j}$ on Free $\left(\left(2^{\omega}\right)^{\Gamma_{j}}\right)$ and $*_{i} \Gamma_{i}$ on Free $\left(\left(2^{\omega}\right)^{*_{i} \Gamma_{i}}\right)$

The use of Borel determinacy is necessary to prove Theorems 1.1 and 1.2 as well as the main lemmas from our earlier paper [28]. We show this by proving the following reversal:

Theorem 1.3. Theorems 1.1] and 1.2 as well as Lemmas 2.1 and 3.12 from [28] are equivalent to Borel determinacy over the base theory $\mathrm{Z}^{-}+\Sigma_{1}-$ replacement + DC.

In fact, the reversal of all these theorems requires only one of their simplest nontrivial cases: when there are two groups in the free product which are both copies of $\mathbb{Z}$. It remains an open question whether any of the consequence of these theorems-both in the current paper and in [28]-require the use of Borel determinacy in their proofs 11

In Section 3, we turn to questions of uniformity in the study of universal countable Borel equivalence relations. We work here in the setting where a countable Borel equivalence relation $E$ on a standard Borel space $X$ is given together with some fixed family of partial Borel functions $\left\{\varphi_{i}\right\}_{i \in \omega}$ on $X$ that generate $E$, for which we use the notation $E_{\left\{\varphi_{i}\right\}}$. In this context, we say that a homomorphism $f$ between $E_{\left\{\varphi_{i}\right\}}$ and $E_{\left\{\theta_{i}\right\}}$ is uniform if a witness that $x E_{\left\{\varphi_{i}\right\}} y$ (that is, a pair of indices $(i, j)$ so that $\varphi_{i}(x)=y$ and

\footnotetext{
${ }^{1}$ Sherwood Hachtman has resolved these questions. See the footnote before Question 2.17
} 
$\left.\varphi_{j}(y)=x\right)$ can be transformed into a witness that $f(x) E_{\left\{\theta_{i}\right\}} f(y)$ in a way that is independent of $x$ and $y$ (see Section 3.1 for a precise definition). If these equivalence relations $E_{\left\{\varphi_{i}\right\}}$ and $E_{\left\{\theta_{i}\right\}}$ are generated by free actions of countable groups, this is equivalent to the assertion that the cocycle (see [16. Appendix B]) associated to $f$ is group homomorphism.

This sort of uniformity arises in the study of Martin's conjecture, where Slaman and Steel have shown that Martin's conjecture for Borel functions is equivalent to the statement that every homomorphism from Turing equivalence to itself is equivalent to a uniform homomorphism on a Turing cone [38]. This idea also arises often in proving nonreducibility results between countable Borel equivalence relations; first we analyze the class of homomorphisms which are uniform on some "large" (e.g. conull) set, and then prove that this analysis is complete by showing every homomorphism is equivalent to a uniform homomorphism on a large set. For instance, this is a typical proof strategy in applications of cocycle superrigidity to the field of countable Borel equivalence relations. See for example [1, 16, 39, 41, 46].

One of the central concepts we study is the idea of uniform universality for countable Borel equivalence relations, which was introduced in unpublished work by Montalbán, Reimann and Slaman. Precisely, $E_{\left\{\varphi_{i}\right\}}$ is said to be uniformly universal (with respect to $\left\{\varphi_{i}\right\}_{i \in \omega}$ ) if there is a uniform reduction of every countable Borel equivalence relation, presented as $E_{\left\{\theta_{i}\right\}}$, to $E_{\left\{\varphi_{i}\right\}}$. Certainly, all known universal countable Borel equivalence relations are uniformly universal with respect to the way they are usually generated, so it is fair to say that the uniformly universal countable Borel equivalence relations include all countable Borel equivalence relations that we can hope to prove universal without dramatically new techniques. We make the following stronger conjecture (see Conjecture 3.3):

Conjecture 1.4. A countable Borel equivalence relation is universal if and only if it is uniformly universal with respect to every way it can be generated.

One attraction of the notion of uniform universality is that we are able to settle many open questions about universality with this additional uniformity assumption, and we can also prove precise characterizations of what equivalence relations are uniformly universal in many settings. The bulk of Section 3 is devoted to theorems of these sorts, and we outline some of our main results:

Theorem 1.5 (Properties of uniform universality).

1. For every countable group $\Gamma$, there is a Borel action of $\Gamma$ generating a 
uniformly universal countable Borel equivalence relation if and only if $\Gamma$ contains a copy of $\mathbb{F}_{2}$, the free group on two generators.

2. Given any uniformly universal countable Borel equivalence relation $E_{\left\{\varphi_{i}\right\}}$ on a standard probability space $(X, \mu)$, there is a $\mu$-conull set A for which $E_{\left\{\varphi_{i}\right\}} \uparrow A$ is not uniformly universal.

3. Both the uniformly universal and non-uniformly universal countable Borel equivalence relations are cofinal under $\subseteq$.

4. An increasing union of non-uniformly universal countable Borel equivalence relations is not uniformly universal. An increasing union of uniformly universal countable Borel equivalence relations need not be uniformly universal.

Theorem 1.6 (Classifications).

1. If $\Gamma$ is a countable group, then the shift action of $\Gamma$ on $2^{\Gamma}$ generates a uniformly universal countable Borel equivalence relation if and only if $\Gamma$ contains a subgroup isomorphic to $\mathbb{F}_{2}$.

2. If $\Gamma$ is a countable group, then the conjugacy action of $\Gamma$ on its subgroups is uniformly universal if and only if $\Gamma$ contains a subgroup isomorphic to $\mathbb{F}_{2}$.

3. (Joint with Jay Williams) If $G$ is a countable subgroup of $S_{\infty}$ and $X$ is a standard Borel space of cardinality at least 3, then the permutation action of $G$ on $X^{\omega}$ is uniformly universal if and only if there exists some $n \in \omega$ and a subgroup $H \leq G$ isomorphic to $\mathbb{F}_{2}$ such that the map $H \rightarrow \omega$ given by $h \mapsto h(n)$ is injective.

4. For every additively indecomposable $\alpha<\omega_{1}$, define the equivalence relation $\equiv_{(<\alpha)}$ on $2^{\omega}$ by $x \equiv_{(<\alpha)}$ y if there exists $\beta<\alpha$ such that $x^{(\beta)} \geq_{T} y$ and $y^{(\beta)} \geq_{T} x$. Then $\equiv_{(<\alpha)}$ is uniformly universal if and only if there is a $\beta<\alpha$ such that $\beta \cdot \omega=\alpha$.

5. If $E_{\left\{\varphi_{i}\right\}_{i \in \omega}}$ is a countable Borel equivalence relation on $2^{\omega}$ coarser than recursive isomorphism and closed under countable uniform joins, then $E_{\left\{\varphi_{i}\right\}_{i \in \omega}}$ is not uniformly universal. (This includes many-one equivalence, tt equivalence, wtt equivalence, Turing equivalence, enumeration equivalence, etc.). Further, this result is not true when $2^{\omega}$ is replaced by $3^{\omega}$. 
Note that several of these results settle open questions about universal equivalence relations in our more restrictive uniform context. For example, Hjorth [2, Question 1.4] [17, Question 6.5.(A)] has asked if $E$ and $F$ are countable Borel equivalence relations, $E$ is universal, and $E \subseteq F$, must $F$ be universal? This is addressed by part (3) of Theorem 1.5. Thomas 41, Question 3.22] has asked whether every universal countable Borel equivalence relation on a standard probability space is non-universal on a conull set. This is addressed by part (2) of Theorem 1.5. Finally, Thomas has conjectured [42, Conjecture 1.5] that free Burnside groups of sufficiently high rank admit Borel actions generating universal countable Borel equivalence relations. This contradicts part (1) of Theorem 1.5, when combined with Conjecture 1.4.

Many of these abstract properties of uniformly universal equivalence relations hinge upon an analysis of equivalence relations from computability theory (some of which are mentioned in the second theorem we have stated above). Of course, the investigation of the universality of equivalence relations from computability theory is interesting in its own right. For example, whether Turing equivalence is a universal countable Borel equivalence relation is a long open question of Kechris [21, and is closely connected with Martin's conjecture on Turing invariant functions, as discussed in [30] and [6].

Another important example of a computability-theoretic equivalence relation is that of recursive isomorphism. Suppose $Z$ is a countable set and $G$ is a group of permutations of $Z$. Then the permutation action of $G$ on $Y^{Z}$ is defined by $(g \cdot y)(z)=y\left(g^{-1}(z)\right)$ for $g \in G, y \in Y^{Z}$, and $z \in Z$. Now the equivalence relation of recursive isomorphism on $Y^{\omega}$ is defined to be the orbit equivalence relation of the permutation action of the group of computable bijections of $\omega$ on $Y^{\omega}$. The universality of recursive isomorphism on $2^{\omega}$ is a long open question. However, several partial results are known: Dougherty and Kechris have shown that recursive isomorphism on $\omega^{\omega}$ is universal [5, 6], which was later improved to $5^{\omega}$ by Andretta, Camerlo, and Hjorth [2]. We further improve this result to $3^{\omega}$ :

Theorem 1.7. Recursive isomorphism on $3^{\omega}$ is a universal countable Borel equivalence relation.

This argument hinges on reducing the problem of universality to a combinatorial problem involving Borel colorings of a family of 2-regular Borel graphs. The problem can be solved if we are working on $3^{\omega}$, however on $2^{\omega}$, the problem can not be solved, as we demonstrate using our new 
game-theoretic tools (see Theorem 3.10 ). Indeed, the games of this paper and of [28] were originally developed specifically to solve this problem. We also restate this combinatorial problem in computability-theoretic language, showing that it is equivalent to an inability to control the computational power of countable uniform joins in a seemingly simple context (see Lemma (3.9). These results are the basis of many of our results mentioned above giving families of equivalence relations which are not uniformly universal.

Now while this combinatorial problem related to the universality of recursive isomorphism on $2^{\omega}$ can not be solved in general, it turns out that it can be solved modulo a nullset with respect to any Borel probability measure. This leads to the following theorem, and part (2) of Theorem 1.5. Recall that a countable Borel equivalence relation $E$ is said to be measure universal if given any Borel equivalence relation $F$ on a standard probability space $(X, \mu)$, there is a Borel $\mu$-conull set $A$ such that $F \uparrow A$ can be Borel reduced to $E$.

Theorem 1.8. Recursive isomorphism on $2^{\omega}$ is a measure universal countable Borel equivalence relation.

Hence, measure-theoretic tools can not be used to show that recursive isomorphism on $2^{\omega}$ is not universal.

Though it remains open whether recursive isomorphism is uniformly universal, we can rule out a large class of uniform proofs based on the close connection between recursive isomorphism and many-one equivalence. Thus, we conjecture the following:

Conjecture 1.9. Recursive isomorphism on $2^{\omega}$ is not a universal countable Borel equivalence relation.

If this conjecture is true, it implies the existence of a pair of Borel equivalence relations of different Borel cardinalities $\left(E_{\infty}\right.$ and recursive isomorphism), for which we can not prove this fact using measure-theoretic tools. Conjecture 1.9 also implies that Turing equivalence is not universal, since it is Borel reducible to recursive isomorphism via the Turing jump.

One of the motivations of this paper is the search for new tools with which to study countable Borel equivalence relations. Currently, measure theoretic methods are the only known way of proving nonreducibility among countable Borel equivalence relations of complexity greater than $E_{0}$ (see Question 4.2). However, many important open problems in the subject are known to be resistant to measure-theoretic arguments. For example, it is 
open whether an increasing union of hyperfinite Borel equivalence relations is hyperfinite [4], and whether recursive isomorphism on $2^{\omega}$ is a universal countable Borel equivalence relation [6]. However, an increasing union of hyperfinite Borel equivalence relations is hyperfinite modulo a nullset with respect to any Borel probability measure by a theorem of Dye and Kreiger [7, 8, 25], and every countable Borel equivalence relation can be embedded into recursive isomorphism on $2^{\omega}$ modulo a nullset by Theorem 3.23 of this paper.

One promising candidate for such a new, non-measure-theoretic tool is Martin's ultrafilter on the Turing-invariant sets. Martin's ultrafilter is sometimes called Martin measure. However, because we will discuss Borel probability measures often in this paper, we will use the terminology of ultrafilters to keep this distinction clear. Martin has conjectured a complete classification of the homomorphisms from Turing equivalence to itself with respect to this ultrafilter which would have many consequences for the study of countable Borel equivalence relations. See [30] for a survey of connections between Martin's conjecture and countable Borel equivalence relations. Many of the results discussed there are due to Simon Thomas, who first recognized the tremendous variety of consequences of Martin's conjecture for the field of countable Borel equivalence relations, beyond just the non-universality of Turing equivalence.

In Section 4, we prove several structure theorems for certain countable Borel equivalence relations using ultrafilters related to Martin's ultrafilter, but which are defined on the quotient of equivalence relations finer than Turing equivalence. To begin, we generalize results from [30], where we showed that $E_{\infty}$ is not a smooth disjoint union of Borel equivalence relations of smaller Borel cardinality and that $E_{\infty}$ achieves its universality on a nullset with respect to any Borel probability measure (which answered questions of Thomas [41, Question 3.20] and Jackson, Kechris, and Louveau [17, Question 6.5.(C)]). We show that these results are all true with $E_{\infty}$ replaced with a larger class of universal structurable Borel equivalence relations which also includes, for example, the universal treeable countable Borel equivalence relation, and the equivalence relation of isomorphism of contractible simplicial complexes.

Our proof of these results uses our games to define a $\sigma$-complete ultrafilter $U$ on the $\sigma$-algebra of Borel $E$-invariant sets for each of these equivalence relations $E$. These ultrafilters are very closely related to Martin's ultrafilter; the equivalence relations $E$ we consider are all subsets of Turing equivalence, and our new ultrafilters agree with Martin's ultrafilter when restricted to Turing invariant sets. Further, these ultrafilters have structure-preserving properties reminiscent of Martin's ultrafilter; if $A \in U$, then $E \leq_{B} E \uparrow A$ 
(i.e. $U$ preserves the Borel cardinality of $E$ ).

We briefly describe the class of equivalence relations for which we obtain these ultrafilters. Suppose $\mathcal{K}$ is a Borel class of countable structures closed under isomorphism. Then a countable Borel equivalence relation $E$ is said to be $\mathcal{K}$-structurable if there is a Borel way of assigning a structure from $\mathcal{K}$ to every $E$-class whose universe is that $E$-class. This notion was defined by Jackson, Kechris and Louveau in [17, and Ben Miller has pointed out that their ideas can be used to show that for every such $\mathcal{K}$, there is a universal $\mathcal{K}$ structurable countable Borel equivalence relation $E_{\infty \mathcal{K}}$ (see Theorem 4.13). It is these equivalence relations for which we obtain our ultrafilters, under the assumption of one more condition: that the class of $\mathcal{K}$-structurable equivalence relations is closed under independent joins (see Section 4.3 for a definition). We now state our result precisely (see Theorems 4.14, 4.15, and 4.16).

Theorem 1.10. Suppose $\mathcal{K}$ is a Borel class of countable structures closed under isomorphism, and let $E_{\infty \mathcal{K}}$ be the universal $\mathcal{K}$-structurable equivalence relation on the space $Y_{\infty \mathcal{K}}$. Then

1. If $\mu$ is a Borel probability measure on $Y_{\infty \mathcal{K}}$, there is a $\mu$-null Borel set $A$ so that $E_{\infty \mathcal{K}} \sqsubseteq_{B}^{i} E_{\infty \mathcal{K}} \uparrow A$.

2. If the class of $\mathcal{K}$-structurable countable Borel equivalence relations is closed under binary independent joins, then there is a Borel cardinality preserving ultrafilter on the quotient space of $E_{\infty \mathcal{K}}$ and hence $E_{\infty \mathcal{K}}$ is not a smooth disjoint union of equivalence relations of smaller Borel cardinality.

3. If the class of $\mathcal{K}$-structurable countable Borel equivalence relations is closed under countable independent joins, then if $\left\{A_{i}\right\}_{i \in \omega}$ is a Borel partition of $E_{\infty \mathcal{K}}$ into countably many sets, then there exists some $A_{i}$ such that $E_{\infty \mathcal{K}} \sqsubseteq_{B} E_{\infty \mathcal{K}}\left\lceil A_{i}\right.$. It follows that for all countable Borel equivalence relations $F, E_{\infty K} \leq_{B} F$ implies $E_{\infty K} \sqsubseteq_{B} F$.

In a future paper joint with Adam Day, we use the ultrafilter constructed here for the universal treeable countable Borel equivalence relation to prove a strengthening of Slaman and Steel's result from 38 that Martin's conjecture for Borel functions is equivalent to every Turing invariant function being uniform Turing invariant on a pointed perfect set.

We hope that these ultrafilters will continue to be useful tools for studying countable Borel equivalence relations in the future. Because they preserve Borel cardinality, they have the potential for proving much sharper 
theorems than other tools which do not necessarily have this property, such as Borel probability measures. Finally, we remark that equivalence relations of the form $E_{\infty \mathcal{K}}$ appear to be underappreciated as natural examples of countable Borel equivalence relations, and many interesting open questions exist regarding how model-theoretic properties of structures in the class $\mathcal{K}$ influence the complexity of the resulting $E_{\infty \mathrm{K}} 2$.

\subsection{Basic definitions, notation, and conventions}

Throughout we will use $X, Y$, and $Z$ for standard Borel spaces, $x, y$, and $z$ for elements of these spaces, and $A, B$, and $C$ for subsets of standard Borel spaces (which will generally be Borel). We will use $f, g$, and $h$ for functions between standard Borel spaces. If $A$ is a subset of a standard Borel space, we will use $A^{\mathrm{c}}$ to denote its complement.

A Borel equivalence relation on a standard Borel space $X$ is an equivalence relation on $X$ that is Borel as a subset of $X \times X$. We will generally use $E$ and $F$ to denote Borel equivalence relations. If $E$ and $F$ are Borel equivalence relations on the standard Borel spaces $X$ and $Y$, then $f: X \rightarrow Y$ is said to be a homomorphism from $E$ to $F$ if for all $x, y \in X$, we have $x E y \Rightarrow f(x) F f(y)$. We say that $E$ is Borel reducible to $F$, noted $E \leq_{B} F$, if there is a Borel function $f: X \rightarrow Y$ so that for all $x, y \in X$, we have $x E y \Longleftrightarrow f(x) F f(y)$. Such a function induces an injection $\hat{f}: X / E \rightarrow Y / F$. The class of Borel equivalence relations under $\leq_{B}$ has a rich structure that been a major topic of research in descriptive set theory in the past few decades. The field has had remarkable success both in calibrating the difficulty of classification problems of interest to working mathematicians, and also in understanding the abstract structure of the space of all classification problems.

If $E$ is a Borel equivalence relation on the standard Borel space $X$, then $A \subseteq X$ is said to be $E$-invariant if $x \in A$ and $x E y$ implies $y \in A$. If a group $\Gamma$ acts on a space $X$, then we say that $A \subseteq X$ is $\Gamma$-invariant if it is invariant under the orbit equivalence relation of the $\Gamma$ action. If $P$ is a Borel property of elements of $x$, then we will often consider the largest $E$-invariant subset of $X$ possessing this property. Precisely, this is the set of $x$ such that for all $y \in X$ where $y E x, y$ has property $P$.

If $E$ and $F$ are equivalence relations on standard Borel spaces $X$ and $Y$, then a Borel embedding of $E$ into $F$ is an injective Borel reduction from $E$ to $F$. If there is a Borel embedding from $E$ to $F$ we denote this

\footnotetext{
${ }^{2}$ Since a first draft of this paper was circulated, these questions have been investigated by Chen and Kechris 3 ]
} 
by $E \sqsubseteq_{B} F$. An invariant Borel embedding is one whose range is $F$ invariant. If there is an invariant Borel embedding from $E$ to $F$ we denote this by $E \sqsubseteq_{B}^{i} F$.

A Borel equivalence relation is said to be countable if all of its equivalence classes are countable. A countable Borel equivalence relation $E$ is said to be universal if for all countable Borel equivalence relations $F$, we have $F \leq_{B} E$. Universal countable Borel equivalence relations arise naturally in many areas of mathematics. For example, isomorphism of finitely generated groups [43], conformal equivalence of Riemann surfaces [15], and isomorphism of locally finite connected graphs [23] are all universal countable Borel equivalence relations.

If $E$ is a countable Borel equivalence relation on $X$, we will use $\phi, \psi, \theta$ to denote partial Borel functions $X \rightarrow X$ whose graphs are contained in $E$. By a theorem of Feldman and Moore [9], for every countable Borel equivalence relation $E$, there exists countably many Borel involutions $\left\{\phi_{i}\right\}_{i \in \omega}$ of $X$ such that $x E y$ if and only if there exists an $i$ such that $\phi_{i}(x)=y$. Hence, every countable Borel equivalence relation is generated by the Borel action of some countable group.

Throughout, we use $\Gamma$ and $\Delta$ to denote countable groups, which we always assume to be discrete, and we use the lowercase $\alpha, \beta, \gamma, \delta$ for their elements. If $X$ is a standard Borel space, then so is the space $X^{\Gamma}$ of functions from $\Gamma$ to $X$ whose standard Borel structure arises from the product topology. We let $E(\Gamma, X)$ denote the equivalence relation on $X^{\Gamma}$ of orbits of the left shift action where $x E(\Gamma, X) y$ if there is a $\gamma \in \Gamma$ such that $\gamma \cdot x=y$. By [4], if $\Gamma$ contains a subgroup isomorphic to the free group $\mathbb{F}_{2}$ on two generators, and $X$ has cardinality $\geq 2$, then $E(\Gamma, X)$ is a universal countable Borel equivalence relation.

If a group $\Gamma$ acts on a space $X$, then the free part of this action is the set $Y$ of $x \in X$ such that for every nonidentity $\gamma \in \Gamma$, we have $\gamma \cdot x \neq x$. We use the notation $\operatorname{Free}\left(X^{\Gamma}\right)$ to denote the free part of the left shift action of $\Gamma$ on $X^{\Gamma}$. We will also let $F(\Gamma, X)$ denote the restriction of the equivalence relation $E(\Gamma, X)$ to this free part.

A Borel graph on a standard Borel space $X$ is a symmetric irreflexive relation on $X$ that is Borel as a subset of $X \times X$. If $\Gamma$ is a marked countable group (i.e. a group equipped with a generating set) and $X$ is a standard Borel space, then we let $G(\Gamma, X)$ note the graph on $\operatorname{Free}\left(X^{\Gamma}\right)$ where there is an edge between $x$ and $y$ if there is a generator $\gamma$ of $\Gamma$ such that $\gamma \cdot x=y$ or $\gamma \cdot y=x$. Hence, the connected components of $G(\Gamma, Y)$ are the equivalence classes of $F(\Gamma, X)$.

A Borel $n$-coloring of a Borel graph $G$ is a function $f$ from the vertices 
of $G$ to $n$ such that if $x$ and $y$ are adjacent vertices, then $f(x) \neq f(y)$. A fact we use several times is that $G(\mathbb{Z}, 2)$ has no Borel 2-coloring, equipping the additive group $\mathbb{Z}$ with its usual set of $\{1\}$ (see [20]). A graph is said to be $d$-regular if every vertex of the graph has exactly $d$ neighbors. We also have from [20] that every Borel $d$-regular graph has a Borel $(d+1)$-coloring.

Give two reals $x, y \in 2^{\omega}$, the join of $x$ and $y$, noted $x \oplus y$ is defined by setting $(x \oplus y)(2 n)=x(n)$ and $(x \oplus y)(2 n+1)=y(n)$ for all $n$. The join of finitely many reals is defined analogously. If we fix some computable bijection $\langle\cdot, \cdot\rangle: \omega^{2} \rightarrow \omega$, we define the uniform computable join $\bigoplus_{i \in \omega} x_{i}$ of countably many reals $x_{0}, x_{1}, \ldots$ by setting $\bigoplus_{i \in \omega} x_{i}(\langle n, m\rangle)=x_{n}(m)$. If $s \in 2^{<\omega}$ and $x \in 2^{\omega}$, we use $s^{\wedge} x$ to denote the concatenation of $s$ followed by $x$.

We use $x^{\prime}$ to denote the Turing jump of a real $x$. If $\alpha$ is a notation for a computable ordinal, then we let $x^{(\alpha)}$ denote the $\alpha$ th iterate of the Turing jump relative to $x$.

\subsection{Acknowledgments}

Theorems 3.7 and 3.23 are from the author's thesis [26]. The author would like his thesis advisor, Ted Slaman, for many years of wise advice.

The author would also like to thank Clinton Conley, Adam Day, Alekos Kechris, Ben Miller, Jan Reimann, Richard Shore, John Steel, Simon Thomas, Anush Tserunyan, Robin Tucker-Drob, Jay Williams, Hugh Woodin, and Jindřich Zapletal for many helpful conversations. 


\section{Games and equivariant functions}

\subsection{The main game}

In this section we introduce the games which are the main technical tool of this paper. They are the natural generalization of the games in [28] to free products of countably many groups, and we use many of the same ideas as that paper. Throughout this section, we fix $I \leq \omega$ and some countable collection $\left\{\Gamma_{i}\right\}_{i \in I}$ of disjoint countable groups. For each $i \in I$, we also fix a listing $\gamma_{i, 0}, \gamma_{i, 1} \ldots$ of the nonidentity elements of $\Gamma_{i}$. We will often abbre-

viate our indexing for clarity. For example, we write $\left(\prod_{i} 2^{\omega}\right)^{*_{i} \Gamma_{i}}$ instead of $\left(\prod_{i \in I} 2^{\omega}\right)^{*_{i \in I} \Gamma_{i}}$.

Of course, a countable product of copies of $2^{\omega}$ is homeomorphic to $2^{\omega}$. However, throughout this section we will work with the space $\prod_{i} 2^{\omega}$ instead of $2^{\omega}$ to streamline the notation in some of our proofs. We also will not use any particular properties of $2^{\omega}$ in this section, which could be replaced by $\omega^{\omega}$ or even the space 2 (that is, $\prod_{i} 2^{\omega}$ would become $\prod_{i} 2$ ). We use the space $2^{\omega}$ since it will be convenient in Section 4 .

We begin with a definition we use throughout in order to partition $*_{i} \Gamma_{i}$ :

Definition 2.1. A group element $\alpha \in *_{i} \Gamma_{i}$ is called a $\Gamma_{j}$-word if $\alpha$ is not the identity and it begins with an element of $\Gamma_{j}$ as a reduced word.

Thus, the group $*_{i} \Gamma_{i}$ is the disjoint union of the set containing the identity $\{1\}$, and the set of $\Gamma_{j}$-words for each $j \in I$.

Fix any $j \in I$. We will be considering games for building an element $y \in\left(\prod_{i} 2^{\omega}\right)^{*_{i} \Gamma_{i}}$ where player I defines $y$ on $\Gamma_{j}$-words, player II defines $y$ on all other nonidentity group elements, and both players contribute to defining $y$ on the identity. We begin by giving a definition that we use to organize the turns on which the bits of $y(\alpha)$ are defined.

Definition 2.2. We define the turn function $t: *_{i} \Gamma_{i} \rightarrow \omega$ as follows. First we set $t(1)=0$. Then, for each nonidentity element $\alpha$ of the free product $*_{i} \Gamma_{i}$, there is a unique sequence $\left(i_{0}, k_{0}\right), \ldots,\left(i_{n}, k_{n}\right)$ such that $i_{m} \neq i_{m+1}$ for all $m$ and $\alpha=\gamma_{i_{0}, k_{0}} \ldots \gamma_{i_{n}, k_{n}}$. We define $t(\alpha)$ to be the least $l$ such that $i_{m}+m<l$ and $k_{m}+m<l$ for all $m \leq n$.

The key property of this definition is that if $i, k \leq l+1$ and $\alpha$ is not a $\Gamma_{i}$-word, then $t\left(\gamma_{i, k} \alpha\right) \leq l+1$ if and only if $t(\alpha) \leq l$. We also have that for each $l$ there are only finitely many $\alpha$ with $t(\alpha)=l$.

We will also define a partition $\left\{W_{i}\right\}_{i \in I}$ of the set $*_{i} \Gamma_{i} \times I$. 
Definition 2.3. For each $j \in I$, let $W_{j}$ be the set of $(\alpha, i) \in *_{i} \Gamma_{i} \times I$ such that $\alpha$ is a $\Gamma_{j}$-word, or $\alpha=1$ and $i=j$.

We are now ready to define our main game:

Definition 2.4 (The main game). Fix a bijection $\langle\cdot, \cdot\rangle: I \times \omega \rightarrow \omega$. Given any $A \subseteq\left(\prod_{i} 2^{\omega}\right)^{*_{i} \Gamma_{i}}$, and any $j \in I$, we define the following game $G_{j}^{A}$ for producing $y \in\left(\prod_{i} 2^{\omega}\right)^{*_{i} \Gamma_{i}}$. Player I goes first, and on each turn the players alternate defining $y(\alpha)(i)(n)$ for finitely many triples $(\alpha, i, n)$. Player I will determine $y(\alpha)(i)(n)$ if $(\alpha, i) \in W_{j}$ and Player II will determine $y(\alpha)(i)(n)$ otherwise. Finally, the value $y(\alpha)(i)(n)$ will be defined on turn $k$ of the game by the appropriate player if $t(\alpha)+\langle i, n\rangle=k$. Player I wins the game if and only if the $y$ that is produced is not in $A$.

Note that $y(\alpha)(i)$ is an element of $2^{\omega}$ and so $y(\alpha)(i)(n)$ is its $n$th bit. Note also that $t(\alpha)$ is the first turn on which $y(\alpha)(i)(n)$ is defined for some $i$ and $n$, and on turn $t(\alpha)+l$, we have that $y(\alpha)(i)(n)$ is defined for the $l$ th pair $(i, n)$.

Now we prove two key lemmas. The first concerns strategies for player I:

Lemma 2.5. Suppose $\left\{A_{i}\right\}_{i \in I}$ is a partition of $\left(\prod_{i} 2^{\omega}\right)^{*_{i} \Gamma_{i}}$. Then player $I$ can not have a winning strategy in $G_{i}^{A_{i}}$ for every $i \in I$.

Proof. We proceed by contradiction. We claim that from such winning strategies, we could produce a $y$ that was simultaneously a winning outcome of player I's strategy in $G_{i}^{A_{i}}$ for every $i \in I$, and hence $y \notin A_{i}$ for all $i \in I$, contradicting the fact that $\left\{A_{i}\right\}_{i \in I}$ is a partition.

To see this, fix winning strategies for player I in each $G_{i}^{A_{i}}$. Inductively, assume all turns $<k$ of the games $G_{i}^{A_{i}}$ have been played and that we have already defined $y(\alpha)(i)(n)$ for all $(\alpha, i, n)$ where $t(\alpha)+\langle i, n\rangle<k$. Now on turn $k$ of the game, the winning strategies for player I in the games $G_{i}^{A_{i}}$ collectively define $y(\alpha)(i)(n)$ on all $(\alpha, i, n)$ where $t(\alpha)+\langle i, n\rangle=k$. Since the sets $W_{j}$ partition $*_{i} \Gamma_{i} \times I$, there is no inconsistency between any of these moves in different games. This defines $y(\alpha)(i)(n)$ for all $(\alpha, i, n)$ where $t(\alpha)+\langle i, n\rangle<k+1$. We can now move for player II in each of the games $G_{i}^{A_{i}}$ using this information, finishing the $k$ th turn of all these games. This completes the induction.

There is a different way of viewing Lemma 2.5 which the reader may find helpful. One can regard the games $G_{i}^{A_{i}}$ in Definition 2.4 as constituting a single game with countably many players: one player for each $i \in I$. The role 
of player $i$ in this multiplayer game corresponds to the role of player $I$ in the game $G_{i}^{A_{i}}$. That is, in this multiplayer game we are still building an element $y \in\left(\prod_{i} 2^{\omega}\right)^{*_{i} \Gamma_{i}}$, but now player $i$ defines $y(\alpha)(i)$ for all $(\alpha, i) \in W_{i}$. The proof of Lemma 2.4 is essentially checking that the games $G_{i}^{A_{i}}$ fit together in this way.

Now in this multiplayer game, instead of declaring a winner, one of the players is instead declared the loser once play is over. That is, the payoff set for the multiplayer game is a Borel partition $\left\{A_{i}\right\}_{i \in I}$ of $\left(\prod_{i} 2^{\omega}\right)^{*_{i} \Gamma_{i}}$, and player $i$ loses if the $y$ that is created during the game is in $A_{i}$. It is a trivial consequence of Borel determinacy that in such a multiplayer game, there must be some player $i$ so that the remaining players have a strategy to collaborate to make player $i$ lose. (Otherwise, if every player has a strategy to avoid losing, playing these strategies simultaneously yields an outcome of the game not in any element of the partition).

We will make use of following projections from $\left(\prod_{i} 2^{\omega}\right)^{*_{i} \Gamma_{i}}$ to $\left(2^{\omega}\right)^{\Gamma_{i}}$.

Definition 2.6. For each $i \in I$, let $\pi_{i}:\left(\prod_{i} 2^{\omega}\right)^{*_{i} \Gamma_{i}} \rightarrow\left(2^{\omega}\right)^{\Gamma_{i}}$ be the function $\pi_{i}(x)(\gamma)=x(\gamma)(i)$

Note that $\pi_{i}$ is $\Gamma_{i}$-equivariant.

Our second key lemma is a way of combining strategies for player II in the game $G_{i}$. In the alternate way of viewing things described above, since there must be some player $i$ so that the remaining players (which viewed jointly are player II in the game $G_{i}$ ) can collaborate to make player $i$ lose, we are now interested in what can be deduced from the existence of such a strategy.

Note that below we speak of a strategy in the game $G_{j}$ instead of $G_{j}^{A}$ (suppressing the superscript) to emphasize that this lemma does not consider a particular payoff set. Eventually in Lemma 4.9 we will apply this lemma when $s_{\gamma}$ depends on $\gamma$.

Lemma 2.7. Fix a $j \in I$, and suppose that to each element $\gamma$ of $\Gamma_{j}$ we associate a strategy $s_{\gamma}$ for player II in the game $G_{j}$. Then there is a $y \in$ $\left(\prod_{i} 2^{\omega}\right)^{*_{i} \Gamma_{i}}$ such that for all $\gamma \in \Gamma_{j}, \gamma \cdot y$ is an outcome of the game $G_{j}$ where player II uses the strategy $s_{\gamma}$. Further, for every $z \in\left(2^{\omega}\right)^{\Gamma_{j}}$, there is a unique such $y$ so that $\pi_{j}(y)=z$.

Proof. Fix a $z \in\left(2^{\omega}\right)^{\Gamma_{j}}$. For each $\gamma \in \Gamma_{j}$ we will play an instance of the game $G_{j}$ whose outcome will be $\gamma \cdot y$, where the moves for player II are made by the strategy $s_{\gamma}$. We will specify how to move for player I in these games. Indeed, at each turn, there will be a unique move for player I that will satisfy our conditions above. We play these games for all $\gamma \in \Gamma_{j}$ simultaneously. 
If $\gamma \in \Gamma_{j}$ and so $\gamma \cdot y$ is an outcome of a play of $G_{j}$ where player II uses the strategy $s_{\gamma}$, then this strategy determines $(\gamma \cdot y)(\alpha)(i)=y\left(\gamma^{-1} \alpha\right)(i)$ for $(\alpha, i) \notin W_{j}$. So let $V_{\gamma}=\left\{\left(\gamma^{-1} \alpha, i\right):(\alpha, i) \notin W_{j}\right\}$ so that $V_{\gamma}$ is the set of $(\beta, i)$ such that player II determines $y(\beta)(i)$ when they move in the game associated to $\gamma$ whose outcome is $\gamma \cdot y$. Note that the $V_{\gamma}$ are pairwise disjoint. This is because if $\alpha$ is not a $\Gamma_{j}$-word and $\gamma \in \Gamma_{j}$, then $\gamma^{-1} \alpha$ is a reduced word, and so both $\gamma^{-1}$ and $\alpha$ can be uniquely determined from $\gamma^{-1} \alpha$. Hence the strategies for player II in these different games do not interfere with each other.

Inducting on $k$, suppose $(\gamma \cdot y)(\alpha)(i)(n)$ is defined for all $\gamma \in \Gamma_{j}, \alpha \in *_{i} \Gamma_{i}$, and $i, n \in \omega$ such that $t(\alpha)+\langle i, n\rangle<k$, and all moves on turns $<k$ have been played in the games. Suppose $\gamma \in \Gamma_{j}$. We will begin by making the $k$ th move for player I in the game defining $\gamma \cdot y$. First, if $k=\langle j, n\rangle$ for some $n$, we must define $(\gamma \cdot y)(1)(j)(n)=y\left(\gamma^{-1}\right)(j)(n)=z\left(\gamma^{-1}\right)(n)$ to ensure that $\pi_{j}(y)=z$. Next, suppose $\beta$ is a $\Gamma_{j}$-word with $t(\beta) \leq k$ such that we can write $\beta=\gamma_{j, l} \alpha$ for some $l<k$ and $\alpha$ which is not a $\Gamma_{j}$-word such that $t(\alpha)<t(\beta)$. Player I must define $y(\beta)(i)(n)$ on turn $k$ where $\langle i, n\rangle=k-t(\beta)$. Now for all $\gamma \in \Gamma_{j}$, we have $(\gamma \cdot y)\left(\gamma_{j, l} \alpha\right)=y\left(\gamma^{-1} \gamma_{j, l} \alpha\right)=\gamma_{j, l}^{-1} \gamma \cdot y(\alpha)$ where of course $\gamma_{j, l}^{-1} \gamma \in \Gamma_{j}$. Hence, since $t(\alpha)+\langle i, n\rangle\langle t(\beta)+\langle i, n\rangle=k$, we have that $(\gamma \cdot y)\left(\gamma_{j, l} \alpha\right)(i)(j)$ has already been defined by the induction hypothesis. Thus, we must make the $k$ th move for player I in the game associated to $\gamma \cdot y$ using this information. Now player II responds by making their $k$ th move in the games, and so we have played the first $k$ turns of the games, and defined $y(\alpha)(i)(n)$ for all $\alpha \in *_{i} \Gamma_{i}$, and $i, n \in \omega$ such that $t(\alpha)+\langle i, n\rangle \leq k$.

Now we combine the above two lemmas to give the following lemma, which is part of Theorem 1.1.

Lemma 2.8. Suppose $I \leq \omega$ and $\left\{\Gamma_{i}\right\}_{i \in I}$ are countable groups. Let $\left\{A_{i}\right\}_{i \in I}$ be a Borel partition of $\left(\prod_{i} 2^{\omega}\right)^{*_{i} \Gamma_{i}}$. Then there exists some $j \in I$ and an injective continuous function $f:\left(2^{\omega}\right)^{\Gamma_{j}} \rightarrow\left(\prod_{i} 2^{\omega}\right)^{*_{i} \Gamma_{i}}$ that is $\Gamma_{j}$-equivariant with respect to the shift actions and such that $\operatorname{ran}(f) \subseteq A_{j}$.

Proof. By Borel determinacy, either player I or player II has a winning strategy in each game $G_{j}^{A_{j}}$. By Lemma 2.5, player II must win $G_{j}^{A_{j}}$ for some $j$. Fix this $j$, and a winning strategy in this game.

We now define the equivariant continuous function $f: 2^{\omega} \rightarrow\left(\prod_{i} 2^{\omega}\right)^{*_{i} \Gamma_{i}}$. We do this using Lemma 2.7; let $f(x)$ be the unique $y$ such that for all $\gamma \in \Gamma_{j}$, and all $x \in 2^{\omega}$, we have $\pi_{j}(y)=x$, and that $\gamma \cdot y$ is an outcome of the winning strategy for player II in the game $G_{j}^{A_{j}}$. Now $f$ is injective since 
$\pi_{j}(f(x))=x$. The equivariance of $f$ follows from the uniqueness property of Lemma 2.7 and the equivariance of $\pi_{j}$, which implies that $\gamma \cdot f(x)$ and $f(\gamma \cdot x)$ are equal. Finally, since $f(x)$ is a winning outcome of player II's strategy in $G_{j}^{A_{j}}$, we have that $f(x) \in A_{j}$ for all $x$. It is easy to check from the proof of Lemma 2.7 that $f$ is continuous. Roughly, the value of each bit of $f(x)$ depends only on finitely many moves in finitely many games which depend on only finitely many bits of $x$.

Remark 2.9. The proof of Lemma 2.8 shows that $f$ can be chosen such that $\pi_{j}(f(x))=x$.

\subsection{The free part of the shift action}

Our next goal is to prove a version of Lemma 2.8 for the free part of the shift action. To begin, we recall a lemma from [28]. Suppose that $I \leq \omega$ and $\left\{E_{i}\right\}_{i \in I}$ is a collection of at least two equivalence relations on $X$. Then the $E_{i}$ are said to be independent if there does not exist a sequence $x_{0}, x_{1}, \ldots, x_{n}$ of distinct elements of $X$, and $i_{0}, i_{1}, \ldots i_{n} \in \omega$ with $n \geq 2$ such that $i_{j} \neq i_{j+1}$ for $j<n$ and $x_{0} E_{i_{0}} x_{1} E_{i_{1}} x_{2} \ldots x_{n} E_{i_{n}} x_{0}$. The join of the $E_{i}$, denoted $\bigvee_{i \in I} E_{i}$, is the smallest equivalence relation containing all the $E_{i}$. Precisely, $x$ and $y$ are $\bigvee_{i \in I} E_{i}$-related if there is a sequence $x_{0}, x_{1}, \ldots x_{n}$ of elements in $X$ such that $x=x_{0}, y=x_{n}$, and for all $j<n$, we have $x_{j} E_{i} x_{j+1}$ for some $i \in I$. Finally, we say that the $E_{i}$ are everywhere non-independent if for every $\bigvee_{i \in I} E_{i}$ equivalence class $A \subseteq X$, the restrictions of the $E_{i}$ to $A$ are not independent.

Lemma 2.10 ([28, Lemma 2.3]). Suppose that $I \leq \omega$ and $\left\{E_{i}\right\}_{i \in I}$ are countable Borel equivalence relations on a standard Borel space $X$ that are everywhere non-independent. Then there exists a Borel partition $\left\{B_{i}\right\}_{i \in I}$ of $X$ such that for all $i \in I,\left(B_{i}\right)^{c}$ meets every $E_{i}$-class.

We will combine this lemma with one other lemma that we will use to deal with the non-free part of the action.

Lemma 2.11. Suppose that for every $i \in I, X_{i}$ is a $\Gamma_{i}$-invariant Borel subset of $\left(\prod_{i} 2^{\omega}\right)^{*_{i} \Gamma_{i}}$. Let $Y$ be the largest invariant set of $y$ such that $y \in X_{i}$ for all $i \in I$. Then there is a Borel partition $\left\{C_{i}\right\}_{i \in I}$ of the complement of $Y$ such that if $A \subseteq C_{i}$ is $\Gamma_{i}$-invariant, then $A \cap X_{i}=\emptyset$.

Proof. We will define Borel sets $C_{i, n}$ for $i \in I$ and $n \in \omega$ which partition $Y^{\mathrm{c}}$. We will then let $C_{i}=\bigcup_{n \in \omega} C_{i, n}$. If $\delta^{-1} \cdot y \notin X_{i}$, say that the pair $(\delta, i)$ 
witnesses $y \notin Y$. Note that if $(\delta, i)$ witnesses $y \notin Y$, then $\left(\gamma^{-1} \delta, i\right)$ witnesses $\gamma \cdot y \notin Y$.

Let $C_{i, 0}$ be the set of $y \in Y^{\mathrm{c}}$ such that $(1, i)$ witnesses $y \notin Y$ and there is no $j<i$ such that $(1, j)$ witnesses $y \notin Y$. Note that $C_{i, 0}$ does not meet $X_{i}$. For $m>0$, let $C_{i, m}$ be the set of $y \in Y^{\mathrm{c}}$ so that $m$ is the minimal length of a $\delta$ so that some $(\delta, j)$ witnesses $y \notin Y$, and $i$ is least such that such a $\delta$ may be chosen to be a $\Gamma_{i}$-word. Note that by the length of a word $\delta \in *_{i} \Gamma_{i}$, we mean that if $\delta=\gamma_{i_{0}, k_{0}} \ldots \gamma_{i_{n}, k_{n}}$ with $i_{m} \neq i_{m+1}$ for all $m$, then the length of $\delta$ is $n+1$.

So suppose now that $A \subseteq C_{i}$ is $\Gamma_{i}$-invariant, and for a contradiction suppose that $m$ was least such that $\left(A \cap C_{i, m}\right) \cap X_{i}$ is nonempty. Let $y$ be an element of this set and note that $m>0$ since $C_{i, 0}$ does not meet $X_{i}$. Since $m>0$, the associated witness that $y \notin Y$ must be of the form $(\delta, j)$, where $\delta=\gamma_{i_{0}, k_{0}} \ldots \gamma_{i_{n}, k_{n}}$ is a $\Gamma_{i}$-word in reduced form, so $i_{0}=i$. But then $\left(\gamma_{i_{1}, k_{1}} \ldots \gamma_{i_{n}, k_{n}}, j\right)$ witnesses $\gamma_{i_{0}, k_{0}}^{-1} \cdot y \notin Y$, and $\gamma_{i_{0}, k_{0}}^{-1} \cdot y \in A$ since $A$ is $\Gamma_{i}$-invariant. But this implies that $\gamma_{i_{0}, k_{0}}^{-1} \cdot y \in C_{i, m^{\prime}}$ for some $m^{\prime}<m$ since $A \subseteq C_{i}$, and $\gamma_{i_{1}, k_{1}} \ldots \gamma_{i_{n}, k_{n}}$ has length strictly less than $\delta$. This contradicts the minimality of $m$.

We can now prove a version of Lemma 2.8 for the free part of the action:

Lemma 2.12. Suppose $I \leq \omega$ and $\left\{\Gamma_{i}\right\}_{i \in I}$ are countable groups. Let $\left\{A_{i}\right\}_{i \in I}$ be a Borel partition of Free $\left(\left(\prod_{i} 2^{\omega}\right)^{*_{i} \Gamma_{i}}\right)$. Then there exists some $j \in I$ and an injective continuous function $f: \operatorname{Free}\left(\left(2^{\omega}\right)^{\Gamma_{j}}\right) \rightarrow \operatorname{Free}\left(\left(\prod_{i} 2^{\omega}\right)^{*_{i} \Gamma_{i}}\right)$ that is $\Gamma_{j}$-equivariant with respect to the shift actions and such that $\operatorname{ran}(f) \subseteq A_{j}$.

Proof. Our idea is to extend our partition $\left\{A_{i}\right\}_{i \in I}$ to cover the whole space $\left(\prod_{i} 2^{\omega}\right)^{*_{i} \Gamma_{i}}$ in such a way that when we apply Lemma 2.8 to this partition, the resulting function $f$ will have the property that $\operatorname{ran}\left(f\left\lceil\operatorname{Free}\left(\left(2^{\omega}\right)^{\Gamma_{j}}\right)\right) \subseteq\right.$ $A_{j}$.

Let $X_{i}$ be the set of $y \in\left(\prod_{i} 2^{\omega}\right)^{*_{i} \Gamma_{i}}$ on which $\Gamma_{i}$ acts freely. That is, $X_{i}=\left\{y \in\left(\prod_{i} 2^{\omega}\right)^{*_{i} \Gamma_{i}}: \forall 1 \neq \gamma \in \Gamma_{i}(\gamma \cdot y \neq y)\right\}$. Let $Y$ be the largest invariant set of $y$ such that $y \in X_{i}$ for every $i$ and let $\left\{C_{i}\right\}_{i \in I}$ be a Borel partition of the complement of $Y$ as in Lemma 2.11.

Note that Free $\left(\left(\prod_{i} 2^{\omega}\right)^{*_{i} \Gamma_{i}}\right)$ is a subset of $Y$. Let $E_{i}$ be the equivalence relation on $Y$ where $x E_{i} y$ if there exists a $\gamma \in \Gamma_{i}$ such that $\gamma \cdot x=y$. Note that the equivalence relations $\left\{E_{i}\right\}$ are everywhere non-independent on the complement $Y \backslash \operatorname{Free}\left(\left(\prod_{i} 2^{\omega}\right)^{*_{i} \Gamma_{i}}\right)$. So by Lemma 2.10, let $\left\{B_{i}\right\}_{i \in I}$ be a Borel partition of $Y \backslash \operatorname{Free}\left(\left(\prod_{i} 2^{\omega}\right)^{*_{i} \Gamma_{i}}\right)$ so that $B_{i}{ }^{\mathrm{c}}$ meets every $E_{i}$-class.

Let $A_{i}^{\prime}=A_{i} \cup B_{i} \cup C_{i}$, so that $\left\{A_{i}^{\prime}\right\}_{i \in I}$ is a Borel partition of $\left(\prod_{i} 2^{\omega}\right)^{*_{i} \Gamma_{i}}$, and apply Lemma 2.8 to obtain a continuous injective equivariant function 
$f:\left(2^{\omega}\right)^{\Gamma_{j}} \rightarrow A_{j}^{\prime}$

Now $\operatorname{ran}\left(f \uparrow \operatorname{Free}\left(\left(2^{\omega}\right)^{\Gamma_{j}}\right)\right)$ is invariant under the $\Gamma_{j}$ action since $f$ is $\Gamma_{j}$-equivariant. Thus, $\operatorname{ran}\left(f \uparrow \operatorname{Free}\left(\left(2^{\omega}\right)^{\Gamma_{j}}\right)\right)$ does not meet $B_{j}$ (whose complement meets every $E_{j}$-class $)$. Since $f$ is injective we also have that $\operatorname{ran}\left(f\left\lceil\right.\right.$ Free $\left.\left(\left(2^{\omega}\right)^{\Gamma_{j}}\right)\right) \subseteq X_{j}$ and hence $\operatorname{ran}\left(f\left\lceil\operatorname{Free}\left(\left(2^{\omega}\right)^{\Gamma_{j}}\right)\right)\right.$ does not meet $C_{j}$ by Lemma 2.11, Hence, $\operatorname{ran}\left(f \uparrow \operatorname{Free}\left(\left(2^{\omega}\right)^{\Gamma_{j}}\right)\right) \subseteq A_{j}$.

There is an interesting application of this lemma to a fact about complete sections of $F\left(\mathbb{F}_{\omega}, 2^{\omega}\right)$.

Theorem 2.13. Suppose that $A$ is a Borel complete section of $F\left(\mathbb{F}_{\omega}, 2^{\omega}\right)$. Then there is an $x \in \operatorname{Free}\left(\omega^{\mathbb{F}_{\omega}}\right)$ and some subgroup $\Gamma$ of $\mathbb{F}_{\omega}$ so that $\Gamma$ is isomorphic to $\mathbb{F}_{\omega}$ and $\gamma \cdot x \in A$ for every $\gamma \in \Gamma$.

Proof. Let $\gamma_{0}, \gamma_{1}, \ldots$ be an enumeration of all the elements of $\mathbb{F}_{\omega}$, and define $\left\{A_{i}\right\}_{i \in \omega}$ inductively by $A_{i}=\gamma_{i} \cdot A \backslash\left(\cup_{j<i} A_{j}\right)$. Note that the $\left\{A_{i}\right\}_{i \in \omega}$ partition Free $\left(\left(2^{\omega}\right)^{\mathbb{F}_{\omega}}\right)$. Now if we write $\mathbb{F}_{\omega}$ as $\mathbb{F}_{\omega} * \mathbb{F}_{\omega} * \ldots$, and apply Lemma 2.12, then there is some $A_{i}$ and corresponding $i$ th copy of $\mathbb{F}_{\omega}$ such that there is an equivariant injection from $\operatorname{Free}\left(\omega^{\mathbb{F} \omega}\right)$ into $\operatorname{Free}\left(\left(2^{\omega}\right)^{\mathbb{F}_{2} * \mathbb{F}_{2} * \ldots}\right)$ whose range is contained in $\gamma_{i} \cdot A$ for some $i$. Let $\Delta$ be this $i$ th copy of $\mathbb{F}_{\omega}$, and note then that if $y \in \operatorname{ran}(f)$ then since $\delta \cdot y \in \gamma_{i} \cdot A$ for all $\delta \in \Delta$, then letting $x=\gamma_{i}^{-1} \cdot y$ and $\Gamma=\gamma_{i}^{-1} \Delta \gamma_{i}$, we are done. To verify, we check that $\gamma_{i}^{-1} \delta \gamma_{i} \cdot x=$ $\gamma_{i}^{-1} \delta \gamma_{i} \cdot\left(\gamma_{i}^{-1} \cdot y\right)=\gamma_{i}^{-1} \cdot(\delta \cdot y) \in A$ since $\delta \cdot y \in \gamma_{i} \cdot A$.

Remark 2.14. Theorem 2.13 is true when we replace $F\left(\mathbb{F}_{\omega}, 2^{\omega}\right)$ by $F\left(\mathbb{F}_{2}, 2^{\omega}\right)$. This is because there is a subgroup of $\mathbb{F}_{2}$ isomorphic to $\mathbb{F}_{\omega}$ for which we can equivariantly embed $F\left(\mathbb{F}_{\omega}, 2^{\omega}\right)$ into $F\left(\mathbb{F}_{2}, 2^{\omega}\right)$ (see [4]). Having done this, then given any Borel complete section $A$ of $F\left(\mathbb{F}_{2}, 2^{\omega}\right)$, in each equivalence class of $F\left(\mathbb{F}_{2}, 2^{\omega}\right)$ that meets the range of this embedding of $F\left(\mathbb{F}_{\omega}, 2^{\omega}\right)$, we can translate $A$ by the least group element that makes $A$ intersect the range of this embedding to obtain $A^{*}$. Now pull $A^{*}$ back under the embedding and apply Theorem 2.13. Theorem 2.13 is also true when we replace $F\left(\mathbb{F}_{\omega}, 2^{\omega}\right)$ with $F\left(\mathbb{F}_{\omega}, 2\right)$ by [36]. Finally, by results of Section 4 , we can find a $\Gamma$ such that $F\left(\mathbb{F}_{\omega}, 2^{\omega}\right)\lceil\{x: \forall \gamma \in \Gamma(\gamma \cdot x \in A)\}$ has the same Borel cardinality as $F\left(\mathbb{F}_{\omega}, 2^{\omega}\right)$.

There is a general theme here that a complete section of the shift action of $\Gamma$ on Free $\left(\left(2^{\omega}\right)^{\Gamma}\right)$ must have a significant amount of structure. Gao, Jackson, Khrone, and Seward have some other results which fit into this theme, which are currently in preparation. See also [27. 


\subsection{Results in reverse mathematics}

In this section, we show that the lemmas we have proved above giving continuous equivariant functions into Borel partitions of $\left(2^{\omega}\right)^{*_{i} \Gamma_{i}}$ truly require the use of determinacy in their proofs, in the sense that these lemmas imply Borel determinacy. We will also show that the main lemma from [28] implies Borel determinacy. These reversals rely on the following observation:

Lemma 2.15. Suppose $X, Y \in\left\{2,3, \ldots, \omega, 2^{\omega}\right\}$, let $\Gamma=\Delta=\mathbb{Z}$, and suppose $f: \operatorname{Free}\left(X^{\Gamma}\right) \rightarrow Y^{\Gamma * \Delta}$ is a continuous $\Gamma$-equivariant function whose range is not a singleton. Then there is a continuous function $g: 2^{\omega} \rightarrow$ Free $\left(X^{\Gamma}\right)$ such that for all $x \in 2^{\omega}$, we have $f(g(x)) \geq_{T} x$.

We are restricting here to the spaces $\left\{2,3 \ldots, \omega, 2^{\omega}\right\}$ and the groups $\Gamma=\Delta=\mathbb{Z}$ so that we can sensibly talk about computability in the space $Y^{\Gamma * \Delta}$. More generally, the same proof will work for any groups $\Gamma$ and $\Delta$ that contain $\mathbb{Z}$ as a subgroup, provided we choose an appropriate way of identifying $\Gamma * \Delta$ with $\omega$ so that the cosets of $\mathbb{Z}$ are computable.

Proof. We will assume that $X=Y=2^{\omega}$. The proof is similar in the other cases. Note that since $f$ is continuous and $\operatorname{ran}(f)$ is not a singleton, we can find basic clopen neighborhoods $U_{0}, U_{1}$ of Free $\left(X^{\Gamma}\right)$ so that $f\left(U_{0}\right)$ and $f\left(U_{1}\right)$ are disjoint. Furthermore, by refining the basic clopen sets $U_{0}$ and $U_{1}$, we may assume that this disjointness is witnessed in the following strong way: there is a $\delta \in \Gamma * \Delta, i \in \omega$, and $j_{0} \neq j_{1} \in 2$ so that for all $x_{0} \in U_{0}$ and $x_{1} \in U_{1}$,

$$
f\left(x_{0}\right)(\delta)(i)=j_{0} \text { and } f\left(x_{1}\right)(\delta)(i)=j_{1} .
$$

Since $U_{0}$ and $U_{1}$ are basic clopen neighborhoods in $X^{\Gamma}$, there are finite sets $S_{0}, S_{1} \subseteq \mathbb{Z}$ and $s_{i, n} \in 2^{<\omega}$ for $i \in\{0,1\}$ and $n \in S_{i}$ so that $U_{i}=\left\{x \in X^{\mathbb{Z}}\right.$ : $\left.\forall n \in S_{i}: x(n) \supseteq s_{i, n}\right\}$. Choose $k$ larger than twice the absolute value of any element of $S_{0}$ or $S_{1}$ so that $k^{\prime} \cdot U_{0}$ and $U_{1}$ are compatible for any $k^{\prime} \in \mathbb{Z}=\Gamma$ with $\left|k^{\prime}\right| \geq k$.

Our idea is to code $x \in 2^{\omega}$ into $g(x) \in \operatorname{Free}\left(X^{\Gamma}\right)$ by putting $n k \cdot g(x) \in U_{i}$ if and only if $x(n)=i$. Then from the value of $(n k \cdot f(g(x)))(\delta)(i)$ (in particular whether it is $j_{0}$ or $\left.j_{1}\right)$, we will be able to determine whether $n k \cdot g(x) \in U_{0}$ or $n k \cdot g(x) \in U_{1}$ and hence the value of $x(n)$. This coding only constrains our choice of $g(x)(m)$ for $m \geq-k$. Hence, we can define $g(x)(m)$ for $m<-k$ so that the sequence $g(x)(m)$ for $m<-k$ is not periodic. This will ensure that $g(x) \in \operatorname{Free}\left(X^{\Gamma}\right)$. It is clear that we can find a continuous map $g: 2^{\omega} \rightarrow \operatorname{Free}\left(X^{\Gamma}\right)$ with this property. 
Recall that Turing determinacy for a pointclass $\Lambda$ is the statement that every Turing invariant set $A \in \Lambda$ either contains a Turing cone, or its complement contains a Turing cone. Turing determinacy is closely connected to determinacy in general. For example, $\boldsymbol{\Sigma}_{1}^{1}$ Turing determinacy is equivalent to $\boldsymbol{\Sigma}_{1}^{1}$ determinacy by Martin [32] and Harrington [14, and Woodin has shown that Turing determinacy is equivalent to $\mathrm{AD}$ in $L(\mathbb{R})$. For Borel sets, a close analysis of Friedman's work in [10] shows that over $\mathrm{Z}^{-}+\Sigma_{1}-$ replacement + DC, Borel Turing determinacy implies Borel determinacy (see [35], [33, Exercises 2.3.6-2.3.11], and [12]).

Theorem 2.16. The following theorems are each equivalent to Borel determinacy over the base theory $\mathrm{Z}^{-}+\Sigma_{1}-$ replacement $+\mathrm{DC}$ : Lemmas [2.8 and 2.12 and hence Theorems 1.1 and 1.2 from this paper, and Lemmas 2.1 and 3.12 from 28 .

Proof. Let $\Gamma=\Delta=\mathbb{Z}$ and suppose $X, Y \in\left\{2,3, \ldots, \omega, 2^{\omega}\right\}$ is appropriate for the theorem or lemma in question which we would like to reverse. In the case of the Lemmas 2.8 and 2.12 in this paper, assume that $I=2$, and $\left\{\Gamma_{i}\right\}_{i \in I}=\{\Gamma, \Delta\}$.

Suppose $A \subseteq Y^{\Gamma * \Delta}$ is Turing invariant. Since Borel Turing determinacy implies Borel determinacy over $\mathrm{Z}^{-}+\Sigma_{1}-$ replacement $+\mathrm{DC}$, it will suffice to prove that $A$ either contains a Turing cone, or is disjoint from a Turing cone. By applying our theorem or lemma, we can find an equivariant continuous map $f$ whose range is contained in $A$ or the complement of $A$. The domain of $f$ will at least include $\operatorname{Free}\left(X^{\Gamma}\right)$, and the codomain will be $Y^{\Gamma * \Delta}$ for some $X, Y \in\left\{2,3, \ldots, \omega, 2^{\omega}\right\}$. Hence by Lemma 2.15, we can find a continuous function $g: 2^{\omega} \rightarrow \operatorname{Free}\left(X^{\Gamma}\right)$ or $g: 2^{\omega} \rightarrow \operatorname{Free}\left(X^{\Delta}\right)$ so that for every $x, f(g(x)) \geq_{T} x$. Now since $f \circ g$ is continuous, on the cone of $x$ above a code for $f \circ g, f(g(x)) \equiv_{T} x$. Hence, the range of $f \circ g$ contains representatives of a Turing cone. Hence either $A$ or its complement contains a cone.

It is very natural to ask about the strength in reverse mathematics of the main theorems in both this paper and [28], since they are proved using these lemmas which reverse to Borel determinacy. These are all open problems 3 We draw attention to a pair of questions which we find particularly interesting.

\footnotetext{
${ }^{3}$ After a preprint of this paper was posted, Sherwood Hachtman resolved these problems. In particular, Questions 2.17 and 2.18 have negative answers. Hachtman's proof uses the fact that all these statements have the following syntactic form: for all Borel functions $f$, there exists a real $x$ such that $R(x, f)$ where $R$ is a Borel condition. Thus, for each $f$, each instance is $\Sigma_{1}^{1}$ in a real code for $f$, and thus these statments are true in
} 
Question 2.17. Does Theorem 3.9 imply Borel determinacy over $\mathrm{Z}^{-}+\Sigma_{1}-$ replacement $+\mathrm{DC}$ ?

Theorem 3.9 stands out to us here because it resembles (at least in a superficial way) Borel diagonalization theorems which are known to have strength in reverse mathematics.

Question 2.18. Does [28, Theorem 3.7] imply Borel determinacy over $\mathrm{Z}^{-}+$ $\Sigma_{1}$ - replacement $+\mathrm{DC}$ ?

Note that by the proof of Theorem [2.16] above, [28, Lemma 2.1] implies Borel determinacy in the case when $\Gamma$ and $\Delta$ contain $\mathbb{Z}$. However, in the case when $\Gamma$ and $\Delta$ are finite, the strength of [28, Lemma 2.1] is open, and it is in fact equivalent to [28, Theorem 3.7]. This is because the spaces Free $\left(\mathbb{N}^{\Gamma}\right)$ and Free $\left(\mathbb{N}^{\Delta}\right)$ are countable, so constructing equivariant functions is trivial once we know $A$ or its complement contain infinitely many $\Gamma$-orbits or $\Delta$-orbits. Note that we know there cannot be an easy measure theoretic or Baire category proof of [28, Theorem 3.7] by [28, Theorem 4.5], except in the trivial case $\Gamma=\Delta=\mathbb{Z} / 2 \mathbb{Z}$. So there is at least some evidence that [28, Theorem 3.7] is hard to prove.

levels of $L$ that are $\Sigma_{1}^{1}$ correct. This includes levels of $L$ that witness the failure of Borel (or even $\Sigma_{4}^{0}$ ) determinacy. 


\section{Uniform universality}

\subsection{Introduction to uniform universality}

In this section, we will investigate a strengthened form of universality for countable Borel equivalence relations. The key idea will be to restrict the class of Borel reductions we consider witnessing $E \leq_{B} F$ to only those reductions $f$ where there is a way of transforming a witness that $x$ and $y$ are $E$-equivalent into a witness that $f(x)$ and $f(y)$ are $F$-equivalent in a way that is independent of $x$ and $y$. To this end, we will begin this section with a discussion of how countable Borel equivalence relations may be generated. Indeed, though the Feldman-Moore theorem [9] implies that every countable Borel equivalence relation can be generated by the Borel action of a countable group, we will prefer to work with a more general way of generating equivalence relations since many equivalence relations from computability theory are not naturally generated by group actions. Such equivalence relations will play a key role in many of the theorems we will prove.

Let $E$ be a countable Borel equivalence relation on a standard Borel space $X$. Then by Lusin-Novikov uniformization [22, Theorem 18.10], there exists a countable set $\left\{\varphi_{i}\right\}_{i \in \omega}$ of partial Borel functions $\varphi_{i}: X \rightarrow X$ such that $x E y$ if and only if there is an $i$ and $j$ such that $\varphi_{i}(x)=y$ and $\varphi_{j}(y)=x$. Conversely, if $X$ is a standard Borel space and $\left\{\varphi_{i}\right\}_{i \in \omega}$ is a countable set of partial Borel functions on $X$ that is closed under composition and includes the identity function, then we define $E_{\left\{\varphi_{i}\right\}}^{X}$ to be the equivalence relation generated by the functions $\left\{\varphi_{i}\right\}_{i \in \omega}$, where $x E_{\left\{\varphi_{i}\right\}}^{X} y$ if there exists an $i$ and $j$ such that $\varphi_{i}(x)=y$ and $\varphi_{j}(y)=x$, in which case we say $x E_{\left\{\varphi_{i}\right\}}^{X} y$ via $(i, j)$. For example, the Turing reductions are a countable set of partial Borel functions on $2^{\omega}$ which generate Turing equivalence.

Our assumption here that the set $\left\{\varphi_{i}\right\}_{i \in \omega}$ is closed under composition is merely a convenience so that we do not have to discuss words in the functions $\left\{\varphi_{i}\right\}_{i \in \omega}$. For this reason, we will assume throughout this section that there is also a computable function $u: \omega^{2} \rightarrow \omega$ such that $\varphi_{i} \circ \varphi_{j}=\varphi_{u(i, j)}$ for all $(i, j) \in \omega^{2}$.

We codify the above into the following convention:

Convention 3.1. Throughout this section, we will let $\left\{\varphi_{i}\right\}_{i \in \omega}$ and $\left\{\theta_{i}\right\}_{i \in \omega}$ denote countable sets of partial functions on some standard Borel space that contain the identity function, and are closed under composition as witnessed by some computable function on indices. We will often omit the 
indexing on $\left\{\varphi_{i}\right\}_{i \in \omega}$ and $\left\{\theta_{i}\right\}_{i \in \omega}$ for clarity. We will let $E_{\left\{\varphi_{i}\right\}}^{X}$ and $E_{\left\{\theta_{i}\right\}}^{Y}$ denote equivalence relations on some standard Borel spaces $X$ and $Y$ that are generated by $\left\{\varphi_{i}\right\}$ and $\left\{\theta_{i}\right\}$ respectively.

Given countable Borel equivalence relations $E_{\left\{\varphi_{i}\right\}}^{X}$ and $E_{\left\{\theta_{i}\right\}}^{Y}$ which are generated by $\left\{\varphi_{i}\right\}$ and $\left\{\theta_{i}\right\}$, say that a homomorphism $f: X \rightarrow Y$ from $E_{\left\{\varphi_{i}\right\}}^{X}$ to $E_{\left\{\theta_{i}\right\}}^{Y}$ is uniform (with respect to $\left\{\varphi_{i}\right\}$ and $\left\{\theta_{i}\right\}$ ) if there exists a function $u: \omega^{2} \rightarrow \omega^{2}$ such that for all $x, y \in X$, if $x E_{\left\{\varphi_{i}\right\}}^{X} y$ via $(i, j)$, then $f(x) E_{\left\{\theta_{i}\right\}}^{Y} f(y)$ via $u(i, j)$, independently of what $x$ and $y$ are.

Now suppose $\Gamma$ is a countable group equipped with a Borel action on a standard Borel space $X$ yielding the countable Borel equivalence relation $E_{\Gamma}^{X}$. In this case we can equivalently regard $E_{\Gamma}^{X}$ as being generated by the functions $x \mapsto \gamma \cdot x$ for each $\gamma \in \Gamma$, and so we can apply our definitions as above in this setting. However, since all of these functions have inverses, the definitions can be simplified a bit. For examples, if $E_{\Delta}^{Y}$ is generated by a Borel action of the countable group $\Delta$ on $Y$ and $f$ is a homomorphism from $E_{\Gamma}^{X}$ to $E_{\Delta}^{Y}$, then $f$ is uniform if and only if there is a function $u: \Gamma \rightarrow \Delta$ such that $u(\gamma) \cdot f(x)=f(\gamma \cdot x)$ for all $\gamma \in \Gamma$. Hence, in the setting where the action of $\Delta$ is free, a homomorphism is uniform if and only if the cocycle associated to it has no dependence on the value of $x \in X$. This type of cocycle superrigidity is well studied and has many applications in the field of Borel equivalence relations, as mentioned in the introduction.

We are ready to give one of the central definitions of this section:

Definition 3.2. A countable Borel equivalence relation $E_{\left\{\varphi_{i}\right\}}^{X}$ generated by $\left\{\varphi_{i}\right\}_{i \in \omega}$ is said to be uniformly universal (with respect to $\left\{\varphi_{i}\right\}_{i \in \omega}$ ) if for every countable Borel equivalence relation $E_{\left\{\theta_{i}\right\}}^{Y}$, there is a Borel reduction $f$ from $E_{\left\{\theta_{i}\right\}}^{Y}$ to $E_{\left\{\varphi_{i}\right\}}^{X}$ that is uniform with respect to $\left\{\theta_{i}\right\}$ and $\left\{\varphi_{i}\right\}$.

The idea of uniform universality was introduced by Montalbán, Reimann, and Slaman (who restricted themselves to the case of equivalence relations generated by Borel actions of countable groups). They showed in unpublished work that Turing equivalence is not uniformly universal with respect to some natural way of generating it by a group. That Turing equivalence is not uniformly universal as it is usually generated with Turing reductions is an easy consequence of Slaman and Steel's work in [38.

Note that when we discuss uniform universality, it is important for us to specify the functions $\left\{\varphi_{i}\right\}_{i \in \omega}$ that we use to generate the equivalence relation $E_{\left\{\varphi_{i}\right\}}^{X}$. In particular, we will show that every universal countable Borel equivalence relation is uniformly universal with respect to some way of generating it (see Proposition 3.7). 
To date, every known universal countable Borel equivalence relation $E$ has been shown to be universal using a proof that is uniform in the sense of Definition 3.2 (and with respect to some natural way of generating $E$ ). Thus, we can regard the class of uniformly universal countable Borel equivalence relations as those which we can hope to prove universal without dramatically new techniques. Indeed, one could make the following ridiculously optimistic conjecture:

Conjecture 3.3. If $E$ is a universal countable Borel equivalence relation, then $E$ is uniformly universal with respect to every way of generating $E$.

This conjecture is attractive since we understand uniformly universality far better than mere universality; Theorems 1.5 and 1.6 from the introduction would settle many open questions about universal countable Borel equivalence relations if Conjecture 3.3 were true.

The various parts of Theorems 1.5 and 1.6 will come from applying some of the tools of Section 2 together with an analysis of some particular examples of natural equivalence relations, many of them from computability theory.

We finish by mentioning that there is another view one could take here which is more computability-theoretic. Instead of studying equivalence relations, we could instead study locally countable quasiorders (which are often called reducibilities). See [44]. If $X$ is a standard Borel space, and $\left\{\varphi_{i}\right\}_{i \in \omega}$ is a countable collection of partial functions on $X$ that contains the identity function and is closed under composition, then we let $\leq_{\left\{\varphi_{i}\right\}}$ be the associated quasiorder where $x \leq_{\left\{\varphi_{i}\right\}} y$ if there exists an $i \in \omega$ such that $\varphi_{i}(y)=x$. In computability theory, there are several important examples of uniform embeddings between natural quasiorders from computability theory. For example, Turing reducibility $\leq_{T}$ embeds into many-one reducibility $\leq_{m}$ via the map $x \mapsto x^{\prime}$, and Turing reducibility also embeds into enumeration reducibility via the map $x \mapsto x \oplus \bar{x}$. These embeddings are uniform in the sense that if $x \leq_{T} y$ via the $i$ th Turing reduction $\varphi_{i}(y)=x$, then there is some many-one reduction/enumeration reduction $\theta_{u(i)}$ depending only on the index $i$ so that the images of $x$ and $y$ are related by $\theta_{u(i)}$. One might want, then, to study the general question of how the usual reducibilities from computability theory are related under uniform Borel embedding/reduction.

All of our proofs in Section 3 work in this context of locally countable quasiorders. So for example, many-one reducibility on $3^{\omega}$ is a uniformly universal locally countable quasiorder (as are poly-time Turing reducibility and arithmetic reducibility by the proofs of [29] and [30]). However, any locally countable Borel quasiorder on $2^{\omega}$ coarser than one-one reducibility 
and closed under countable uniform joins is not a uniformly universal locally countable quasiorder.

\subsection{Basic results on uniform universality}

We will begin by proving some basic facts about uniform universality. Since the composition of two uniform homomorphisms is uniform, it is clear that if $E_{\left\{\varphi_{i}\right\}}^{X}$ is uniformly universal and there is a uniform reduction of $E_{\left\{\varphi_{i}\right\}}^{X}$ to $E_{\left\{\theta_{i}\right\}}^{Y}$, then $E_{\left\{\theta_{i}\right\}}^{Y}$ is also uniformly universal. Hence, to demonstrate that some equivalence relation is uniformly universal, is enough to show that we can uniformly reduce a single uniformly universal equivalence relation to it. For this purpose, we explicitly show below that both $E\left(\mathbb{F}_{\omega}, 2^{\omega}\right)$ and $E\left(\mathbb{F}_{2}, 2^{\omega}\right)$ are uniformly universal.

Proposition 3.4. $E\left(\mathbb{F}_{\omega}, 2^{\omega}\right)$ and $E\left(\mathbb{F}_{2}, 2^{\omega}\right)$ are both uniformly universal (as generated by the left shift actions).

Proof. This proposition simply follows from the proofs of the universality of these equivalence relations given in [4]. We will recapitulate this argument to explicitly demonstrate how these constructions are uniform, and because we will eventually require a careful analysis of its details.

Fix some equivalence relation $E_{\left\{\varphi_{i}\right\}}^{Y}$ generated by $\left\{\varphi_{i}\right\}_{i \in \omega}$ which we wish to uniformly reduce to $E\left(\mathbb{F}_{\omega}, 2^{\omega}\right)$. We may assume that $Y \subsetneq 2^{\omega}$ is a strict subset of $2^{\omega}$, by exploiting the isomorphism theorem for standard Borel spaces. Let $p$ be some distinguished point in $2^{\omega} \backslash Y$, and let $Y^{*}=Y \cup\{p\}$.

Let the countably many generators of the group $\mathbb{F}_{\omega}$ be $\left\{\gamma_{i, j}\right\}_{(i, j) \in \omega^{2}}$, exploiting some bijection between $\omega$ and $\omega^{2}$. For each $(i, j) \in \omega^{2}$ define the function $\theta_{\gamma_{i, j}}: Y^{*} \rightarrow Y^{*}:$

$$
\theta_{\gamma_{(i, j)}}(y)= \begin{cases}\theta_{i}(y) & \text { if } y \in Y \text { and } \theta_{j}\left(\theta_{i}(y)\right)=y \\ p & \text { otherwise }\end{cases}
$$

Define $\theta_{\gamma_{(i, j)}^{-1}}$ to be $\theta_{\gamma_{(j, i)}}$. Finally, we can define $\theta_{w}$ for any reduced word $w \in F_{\omega^{2}}$ by composing the $\theta_{\gamma_{(i, j)}}$ and $\theta_{\gamma_{(i, j)}^{-1}}$ in the obvious way. Let $\theta_{1}$ be the identity function.

We define our reduction $f: Y \rightarrow\left(2^{\omega}\right)^{\mathbb{F}_{\omega}}$ from $E_{\left\{\theta_{i}\right\}}^{Y}$ to $E\left(\mathbb{F}_{\omega}, 2^{\omega}\right)$ by $f(y)(\alpha)=\theta_{\alpha}(y)$. This is a uniform reduction; if $x E_{\left\{\theta_{i}\right\}}^{Y} y$ via $(i, j)$, then $f(x) E\left(\mathbb{F}_{\omega}, 2^{\omega}\right) f(y)$ via the generator $\gamma_{(i, j)}$.

For the case of $E\left(\mathbb{F}_{2}, 2^{\omega}\right)$, let $p$ be a distinguished point in $2^{\omega}$, and let $\rho: \mathbb{F}_{\omega} \rightarrow \mathbb{F}_{2}$ be an embedding of the group $\mathbb{F}_{\omega}$ into $\mathbb{F}_{2}$. Now we define our 
uniform Borel embedding $g:\left(2^{\omega}\right)^{\mathbb{F}_{\omega}} \rightarrow\left(2^{\omega}\right)^{\mathbb{F}_{2}}$ from $E\left(\mathbb{F}_{\omega}, 2^{\omega}\right)$ to $E\left(\mathbb{F}_{2}, 2^{\omega}\right)$ by:

$$
g(x)(\alpha)= \begin{cases}x\left(\rho^{-1}(\alpha)\right) & \text { if } \alpha \in \operatorname{ran}(\rho) \\ p & \text { otherwise }\end{cases}
$$

The uniformity of this reduction is witnessed by $\rho$.

Note that since $E\left(\mathbb{F}_{\omega}, 2^{\omega}\right)$ is generated by a group action, a countable Borel equivalence relation $E_{\left\{\varphi_{i}\right\}}^{X}$ is uniformly universal if and only if $E\left(\mathbb{F}_{\omega}, 2^{\omega}\right)$ is uniformly reducible to $E_{\left\{\varphi_{i}\right\}}^{X}$ if and only if there is a uniform reduction of every equivalence relation generated by a Borel action of a countable group to $E_{\left\{\varphi_{i}\right\}}^{X}$. Hence, our more general definition agrees with the original definition of Montalbán, Reimann, and Slaman if we restrict to the special case of equivalence relations equipped with group actions generating them.

Another useful consequence of Theorem 3.4 is the following, which says that our uniform reductions can always be assumed to have their uniformity witnessed by a computable function.

Lemma 3.5. If $E_{\left\{\varphi_{i}\right\}}^{X}$ is a uniformly universal countable Borel equivalence relation, then for every countable Borel equivalence relation $E_{\left\{\theta_{i}\right\}}^{Y}$, there is a uniform reduction from $E_{\left\{\theta_{i}\right\}}^{Y}$ to $E_{\left\{\varphi_{i}\right\}}^{X}$ with the additional property that its uniformity function $u: \omega^{2} \rightarrow \omega^{2}$ is computable.

Proof. This follows by analyzing the proof of Proposition 3.4 above. First, the reduction of $E_{\left\{\theta_{i}\right\}}^{Y}$ to $E\left(\mathbb{F}_{\omega}, 2^{\omega}\right)$ has a computable uniformity function. Second, there is a computable embedding of $\mathbb{F}_{\omega}$ into $\mathbb{F}_{2}$ (where the $n$th generator of $\mathbb{F}_{\omega}$ is mapped to $\alpha^{n} \beta \alpha^{-n}$ where $\alpha$ and $\beta$ are the two generators of $\mathbb{F}_{2}$ ). Hence, composing these two uniform reductions, we get one from $E_{\left\{\theta_{i}\right\}}^{Y}$ to $E\left(\mathbb{F}_{2}, 2^{\omega}\right)$ with a computable uniformity function.

Finally, take a uniform reduction from $E\left(\mathbb{F}_{2}, 2^{\omega}\right)$ to $E_{\left\{\varphi_{i}\right\}}^{X}$, since $E_{\left\{\varphi_{i}\right\}}^{X}$ is assumed to be uniformly universal. This reduction can also be assumed to have a computable uniformity function since it is enough just to know how the uniformity of the two generators of $\mathbb{F}_{2}$ is witnessed (recall that by Convention [3.1, we are assuming that composition of functions in $\left\{\varphi_{i}\right\}$ is witnessed by a computable function). Now we are done: compose the reduction of $E_{\left\{\theta_{i}\right\}}^{Y}$ to $E\left(\mathbb{F}_{2}, 2^{\omega}\right)$ with the reduction from $E\left(\mathbb{F}_{2}, 2^{\omega}\right)$ to $E_{\left\{\varphi_{i}\right\}}^{X}$.

The above lemma will be used in Theorem 3.13 as part of proving that a large class of equivalence relations are not uniformly universal. 
We note one final consequence of Proposition 3.4.

Proposition 3.6. If $E_{\left\{\phi_{0, i}\right\}_{i \in \omega}}^{X} \subseteq E_{\left\{\phi_{1, i}\right\}_{i \in \omega}}^{X} \ldots$ is an increasing sequence of countable Borel equivalence relations that are not uniformly universal, then their union $E_{\left\{\theta_{i}\right\}}^{X}$ is not uniformly universal, where $\left\{\theta_{i}\right\}$ is the generating family obtained by closing the $\left\{\phi_{j, i}\right\}$ under composition.

Proof. Since $E\left(\mathbb{F}_{2}, 2^{\omega}\right)$ is uniformly universal and $\mathbb{F}_{2}$ is finitely generated, any uniform reduction from $E\left(\mathbb{F}_{2}, 2^{\omega}\right)$ to $E_{\left\{\theta_{i}\right\}}^{X}$ must be contained inside $E_{\left\{\phi_{j, i}\right\}_{i \in \omega}}^{X}$ for some $j$ since the two generators of $\mathbb{F}_{2}$ correspond to two pairs of functions that are from some $\left\{\phi_{i, j}\right\}_{i \in \omega}$.

Hence, uniformly universal equivalence relations are not "approximable from below" by non-uniformly universal equivalence relations. See 40] and 30] for some related results on strong ergodicity that show that under the assumption of Martin's conjecture, the (weakly) universal countable Borel equivalence relations are "much larger" than the non (weakly) universal ones.

Next, we show that every universal countable Borel equivalence relation is uniformly universal with respect to some way of generating it.

Proposition 3.7. If $E$ is a universal countable Borel equivalence relation, then there is some countably family of functions generating $E$ for which it is uniformly universal.

Proof. This is a trivial corollary of [30, Theorem 3.6], that if $E$ is a universal countable Borel equivalence relation, then $F \sqsubseteq_{B} E$, for every countable Borel equivalence relation $F$.

To see this, let $F=E\left(\mathbb{F}_{\omega}, 2^{\omega}\right)$ which is uniformly universal. Then we can embed $E\left(\mathbb{F}_{\omega}, 2^{\omega}\right)$ into $E$ with an injective Borel function $f$. Now take the partial functions generating the image of $E\left(\mathbb{F}_{\omega}, 2^{\omega}\right)$ on $\operatorname{ran}(f)$ and extend these functions to a larger countable set that generates $E$. With respect to this set of generators, $E$ is uniformly universal.

Indeed, by the same argument, there is some group action generating $E$ with respect to which it is uniformly universal.

We will finish this section with a simple application of the results of Section 2 to uniform universality. In particular, we will prove part (1) of Theorem 1.5.

Theorem 3.8. Suppose $\Gamma$ is a countable group. Then there exists a Borel action of $\Gamma$ on a standard Borel space $X$ such that $E_{\Gamma}^{X}$ is uniformly universal if and only if $\Gamma$ contains $\mathbb{F}_{2}$ as a subgroup. 
Proof. We begin with the forward implication. Since $E_{\Gamma}^{X}$ is uniformly universal, there exists a uniform Borel reduction of $F\left(\mathbb{F}_{2} * \mathbb{F}_{2} * \ldots, 2^{\omega}\right)$ to $E_{\Gamma}^{X}$. Now by Lusin-Novikov uniformization [22, Theorem 18.10], we can partition Free $\left(\left(2^{\omega}\right)^{\mathbb{F}_{2} * \mathbb{F}_{2} * \ldots}\right)$ into countably many Borel sets $\left\{A_{i}\right\}_{i \in \omega}$ such that $f$ is injective on each $A_{i}$. Now by Lemma 2.12, let $g$ be an equivariant Borel injection of $F\left(\mathbb{F}_{2}, 2^{\omega}\right)$ into $F\left(\mathbb{F}_{2} * \mathbb{F}_{2} * \ldots, 2^{\omega}\right) \uparrow A_{j}$ for some $j$ (i.e. equivariant for the $i$ th copy of $\mathbb{F}_{2}$ ). Then $f \circ g$ is a uniform injective Borel homomorphism from $F\left(\mathbb{F}_{2}, 2^{\omega}\right)$ to $E_{\Gamma}^{X}$. If $u: \mathbb{F}_{2} \rightarrow \Gamma$ witnesses this uniformity, then it is clear that the image of the two generators of $\mathbb{F}_{2}$ under $u$ generates a copy of $\mathbb{F}_{2}$ inside $\Gamma$, since $f \circ g$ is injective.

The reverse implication follows from the fact that if $\Gamma$ contains $\mathbb{F}_{2}$ as a subgroup, then $E\left(\Gamma, 2^{\omega}\right)$ is uniformly universal by [4]. (Following essentially the same argument as that in Proposition 3.4.)

Thomas has previously considered the question of which countable groups admit Borel actions that generate universal countable Borel equivalence relations [42. From our uniform perspective, Theorem 3.8 gives a complete answer to this question. Note that the theorem above combined with Conjecture 3.3 contradicts Thomas' Conjecture [42, Conjecture 1.5] that Burnside groups of sufficiently high exponent can generate universal countable Borel equivalence relations.

Another corollary of Theorem 3.8 is a classification of which countable groups $\Gamma$ generate uniformly universal countable Borel equivalence relations with their shift actions on $2^{\Gamma}$ and their conjugacy actions on their subgroups. If $\mathbb{F}_{2} \leq \Gamma$, then both these actions are uniformly universal by [4] and [2]. Theorem 3.8 implies the converses of these two theorems are true. Hence, we have parts (1) and (2) of Theorem 1.6.

\subsection{Limitations on controlling countable joins}

In this section we will show how results from Section 2 can be used to infer a limitation on our ability to control the computational power of countable uniform joins, which we will then use to prove that a number of equivalence relations from computability theory are not uniformly universal.

The general problem of controlling the computational power of finite and countable joins is a frequent topic of investigation in computability theory. To introduce our lemma let us first recall two contrasting pieces of folklore.

First, suppose $\leq_{P}$ is a Borel quasiorder on $2^{\omega}$ with meager sections (e.g. a quasiorder such that for every $y,\left\{x: x \leq_{P} y\right\}$ is countable). Then there is a continuous injection $f: 2^{\omega} \rightarrow 2^{\omega}$ such for every $x \in 2^{\omega}$ and every finite 
sequence of $y_{0}, y_{1}, \ldots y_{n} \in X$ not containing $x$, we have

$$
f(x) \not_{P} f\left(y_{0}\right) \oplus \ldots \oplus f\left(y_{n}\right)
$$

This is easy to show using a simple Baire category argument (see for instance the Kuratowski-Mycielski theorem [22, Theorem 19.1]). In computability theory, $\operatorname{ran}(f)$ is called an independent set for $\leq_{P}$.

Second, suppose we consider countable joins instead of finite joins. Then the analogue of the above fact becomes false. Let us consider one-onereducibility here for concreteness, where $x \leq_{1} y$ if there is a computable injection $\rho: \omega \rightarrow \omega$ such that $x(n)=y(\rho(n))$. Now if $f: 2^{\omega} \rightarrow 2^{\omega}$ is any (not necessarily Borel) function, then there must exist $x \in 2^{\omega}$ and a countable sequence $y_{0}, y_{1}, \ldots \in 2^{\omega}$ not containing $x$ such that

$$
f(x) \leq_{1} f\left(y_{0}\right) \oplus f\left(y_{1}\right) \oplus \ldots
$$

To see this, we may clearly assume $f$ is injective (else the statement is trivial). But then there must be an $n$ such that there are infinitely many $y$ with $f(y)(n)=0$ and infinitely many $y$ such that $f(y)(n)=1$. Thus, by taking the union of two such countably infinite sets of $y$ and permuting this set, we can code any real into the sequence $f\left(y_{0}\right)(n), f\left(y_{1}\right)(n), \ldots$

We now prove a substantial strengthening of this second fact of folklore for Borel functions $f$. Suppose that instead of arbitrary countable joins, we have the dramatically more modest goal of controlling for each $x$ the countable join of a single sequence of $y_{i}$ that depends in a Borel way on $x$. It turns out that this too is impossible!

Theorem 3.9. Let $\mathbb{F}_{\omega}$ be the free group on the $\omega$ many generators $\gamma_{0}, \gamma_{1}, \ldots$ and let $X=$ Free $\left(\left(2^{\omega}\right)^{\mathbb{F}_{\omega}}\right)$. To each $x \in X$ we associate the single countable sequence $\gamma_{0} \cdot x, \gamma_{1} \cdot x, \ldots$ which does not include $x$.

Then for all Borel functions $f: X \rightarrow 2^{\omega}$, there exists an $x \in X$ such that

$$
f(x) \leq_{1} f\left(\gamma_{0} \cdot x\right) \oplus f\left(\gamma_{1} \cdot x\right) \oplus \ldots
$$

Indeed, there is an $x$ such that $f(x)(i)=f\left(\gamma_{i} \cdot x\right)(i)$ for all $i \in \omega$.

Proof. Let $f$ be any Borel function from $X$ to $2^{\omega}$, and let $A_{i}$ be the set of $x \in X$ such that $i$ is the least element of $\omega$ such that $f(x)(i) \neq f\left(\gamma_{i}\right.$. $x)(i)$. Assume for a contradiction that the sets $A_{i}$ partition $X$. Then by Lemma 2.12 there exists some $i$ such that there is a $\left\langle\gamma_{i}\right\rangle$-equivariant Borel injection $h$ from Free $\left(\left(2^{\omega}\right)^{\left\langle\gamma_{i}\right\rangle}\right)$ to $X$ such that $\operatorname{ran}(h) \subseteq A_{i}$. Note then that for all $x$, we have $f(h(x))(i) \neq f\left(h\left(\gamma_{i} \cdot x\right)\right)(i)$. But $x \mapsto f(h(x))(i)$ would then give a Borel 2 -coloring of the graph $G\left(\mathbb{Z}, 2^{\omega}\right)$. This is easily seen to be impossible with an ergodicity argument. (See [20]). 
Of course $\left(2^{\omega}\right)^{\mathbb{F}_{\omega}}$ is homeomorphic to $2^{\omega}$, and so we could regard $X$ above as a subset of $2^{\omega}$.

Now it turns out that controlling countable joins in the way shown to be impossible by Theorem 3.9 shows up as a subproblem in many natural constructions aimed at showing certain equivalence relations from computability theory are uniformly universal. Our next goal will be to pivot Theorem 3.9 and these failed constructions into a proof that many equivalence relations from computability theory are not uniformly universal.

Before we finish, we make one more remark: the lemma above can be restated in graph-theoretic language:

Theorem 3.10. There is a standard Borel space $X$ and countably many 2-regular Borel graphs $\left\{G_{i}\right\}_{i \in \omega}$ on $X$ such that for every Borel set $\left\{c_{i}\right\}_{i \in \omega}$ where $c_{i}$ is a Borel 3-coloring of $G_{i}$, there is an $x \in X$ such that $c_{i}(x)=c_{j}(x)$ for all $i, j$ (i.e. $x$ is $\left\{c_{i}\right\}$-monochromatic).

Proof. Let $X=\operatorname{Free}\left(\left(2^{\omega}\right)^{\mathbb{F}_{\omega}}\right)$ and $G_{i}$ be the graph generated by $g_{i}$ as in Theorem 3.9. Then given countably many 3 -colorings $c_{i}$, let $f: X \rightarrow 2^{\omega}$ be defined by $f(x)(i)=c_{i}(x)$ if $c_{i}(x) \in\{0,1\}$ and $f(x)(i)=c_{i}\left(g_{i}(x)\right)$ if $c_{i}(x) \notin\{0,1\}$. Now apply Theorem 3.9 .

We mention this restatement largely for a historical reason: in [26], we showed that the above coloring problem was equivalent to the uniform universality of many-one equivalence. The proof of the forward direction of this result is essentially contained in the proof of Theorem 3.23. The proof of the converse of this theorem makes essential use of a notion of forcing due to Conley and Miller. We refer the interested reader to [26, Section 5.2]. The equivalence of this coloring problem with the uniform universality of many-one equivalence was the catalyst that led the games studied in this paper and thus most of our results, as well as the results of [28].

\subsection{Uniform universality and equivalence relations from com- putability theory}

In this section, we will show that a large class of equivalence relations from computability theory are not uniformly universal. Beyond the inherent interest in classifying such equivalence relations our analysis will also be used to prove the remaining parts of Theorem 1.5. We begin with a simple lemma.

Lemma 3.11. Suppose $x, y_{0}, y_{1}, \ldots \in 2^{\omega}$ are each of the form $\oplus_{i \in \omega} z$ for some $z \in 2^{\omega}$, and there is a computable function $u: \omega \rightarrow \omega$ such that $x \equiv_{1} y_{i}$ 
via the program $u(i)$ for all $i \in \omega$. Then there exists a pair of computable bijections $r, s: \omega \rightarrow \omega$ such that $x(r(n))=y_{s(n)}(n)$ for all $n$.

Proof. The point of this lemma is that $x$ looks like a "diagonal" of the join of the $y_{i}$ after permuting $x$ by $r$, and the $y_{i}$ by $s$.

Our argument is a simple back and forth construction. At each stage of the construction we will have defined $r$ and $s$ on the same finite domain.

At even steps, we begin by picking the least $n$ not in the domain of $r$ and $s$. Let $m$ be the least number not in the range of $s$, and define $s(n)=m$. Now since $x \equiv_{1} y_{m}$, we have that $y_{m}(n)=x(\langle i, j\rangle)$ for some $\langle i, j\rangle \in \omega$. It is possible that $\langle i, j\rangle$ is already in the range of $r$ but we can always find some $\left\langle i^{*}, j\right\rangle$ not already in the range of $r$, and set $r(n)=\left\langle i^{*}, j\right\rangle$, since $y_{m}(\langle i, j\rangle)=y_{m}\left(\left\langle i^{*}, j\right\rangle\right)$ since $y_{m}$ is of the form $\oplus_{i \in \omega} z$ for some $z \in 2^{\omega}$.

At odd steps, we pick the least $k$ and $m$ that are not in the range of $r$ and $s$ respectively. Then since $x$ is recursively isomorphic to $y_{m}$, there is some $\langle i, j\rangle$ such that $x(k)=y_{m}(\langle i, j\rangle)$. Again, we can find some $\left\langle i^{*}, j\right\rangle$ not already in the domain of $r$ and $s$ and set $r\left(\left\langle i^{*}, j\right\rangle\right)=k$ and $s\left(\left\langle i^{*}, j\right\rangle\right)=m$.

It is a fundamental property of Turing reducibility that if we computably specify countably many Turing reductions, then we can run them all simultaneously to produce the uniform join of their outputs. It is this idea which we encapsulate into our next definition:

Definition 3.12. Suppose that $E_{\left\{\varphi_{i}\right\}}^{2^{\omega}}$ is a countable Borel equivalence relation on $2^{\omega}$ generated by $\left\{\varphi_{i}\right\}_{i \in \omega}$. Say that $E$ is closed under countable uniform joins if whenever $x, y_{0}, y_{1}, \ldots \in 2^{\omega}, u: \omega \rightarrow \omega^{2}$ is computable, and $x E y_{i}$ via $u(i)$ for all $i \in \omega$, then $x E \bigoplus_{i \in \omega} y_{i}$.

For example, many-one equivalence, $t t$ equivalence, Turing equivalence, and enumeration equivalence all have this property, as generated by their usual family of reductions.

Theorem 3.13. Suppose that $E_{\left\{\varphi_{i}\right\}}$ is a countable Borel equivalence relation on $2^{\omega}$ that is coarser than recursive isomorphism and is closed under countable uniform joins. Then $E_{\left\{\varphi_{i}\right\}}$ is not uniformly universal.

Proof. Let $\mathbb{F}_{\omega}=\left\langle\gamma_{i}: i \leq \omega\right\rangle$ be the free group on the generators $\gamma_{0}, \gamma_{1} \ldots, \gamma_{\omega}$. Let $\Gamma$ be the subgroup $\left\langle\gamma_{i} \gamma_{\omega}^{-1}: i<\omega\right\rangle$, which is isomorphic to $\mathbb{F}_{\omega}$. Note that $\gamma_{\omega} \notin \Gamma$. Let $X=\operatorname{Free}\left(\left(2^{\omega}\right)^{F_{\omega}}\right)$, and let $F_{\Gamma} \subseteq F\left(\mathbb{F}_{\omega}, 2^{\omega}\right)$ be the equivalence relation on $X$ where $x F_{\Gamma} y$ if $\alpha \cdot x=y$ for some $\alpha \in \Gamma$. It is interesting to note that $F_{\Gamma}$ is Borel isomorphic to $F\left(\mathbb{F}_{\omega}, 2^{\omega}\right)$ and is hence a universal treeable countable Borel equivalence relation. 
Now for a contradiction, let $f$ be a uniform Borel reduction of $F_{\Gamma}$ to $E_{\left\{\varphi_{i}\right\}}$ with a computable uniformity function by Lemma 3.5. So for all $x \in X$ and $\alpha \in F_{\Gamma}$ we have that $f(x) E f(\alpha \cdot x)$ uniformly in $\alpha$. Let $\alpha_{0}, \alpha_{1}, \ldots$ be a computable listing of all the elements of $\Gamma$ in which each element appears infinitely many times. Let $\hat{f}(x)=\bigoplus_{i \in \omega} f\left(\alpha_{i} \cdot x\right)$. Then we have $f(x) E \hat{f}(x)$ since $E$ is closed under countable uniform joins. It is clear that $\hat{f}\left(\gamma_{\omega} \cdot x\right)$ and $\hat{f}\left(\gamma_{i} \cdot x\right)$ are recursively isomorphic for all $i \in \omega$ by permuting columns. Further, since each group element appears infinitely often in our listing $\left\{\alpha_{i}\right\}_{i \in \omega}$ of $\Gamma$, we can apply Lemma 3.11 to obtain a pair of computable bijections $r, s: \omega \rightarrow \omega$ such that $\hat{f}\left(\gamma_{\omega} \cdot x\right)(r(i))=\hat{f}\left(\gamma_{s(i)} \cdot x\right)(i)$ for all $x \in X$ and all $i \in \omega$.

Now for all $x \in X$, since $\gamma_{\omega} \notin \Gamma$, we have that $x$ E $/ \gamma_{\omega} \cdot x$, and so $f(x) E f\left(\gamma_{\omega} \cdot x\right)$, which implies $\hat{f}(x) E \hat{f}\left(\gamma_{\omega} \cdot x\right)$ and thus $\hat{f}(x)$ and $\hat{f}\left(\gamma_{\omega} \cdot x\right)$ are not recursively isomorphic. Hence, for each $x \in X$, we have that $\hat{f}(x)(i) \neq$ $\hat{f}\left(\gamma_{s(i)}\right)(i)$ for some $i \in \omega$, since otherwise $\hat{f}(x)$ and $\hat{f}\left(\gamma_{\omega} \cdot x\right)$ would be recursively isomorphic via $r$. We will apply the same idea as the proof of Theorem 3.9 to obtain a contradiction.

For each $i \in \omega$, let $A_{s(i)}$ be the set of $x \in X$ such that $i$ is the least element of $\omega$ such that $\hat{f}(x)(i) \neq \hat{f}\left(\gamma_{s(i)} \cdot x\right)(i)$. By Lemma 2.12, there is some $i$ and a $\left\langle\gamma_{s(i)}\right\rangle$-equivariant Borel function $g$ from Free $\left(\left(2^{\omega}\right)^{\left\langle\gamma_{s(i)}\right\rangle}\right)$ to Free $\left(\left(2^{\omega}\right)^{\mathbb{F}_{\omega}}\right)$ such that $\operatorname{ran}(g) \subseteq A_{s(i)}$. Then $x \mapsto \hat{f}(g(x))(i)$ yields a Borel 2-coloring of $G\left(\mathbb{Z}, 2^{\omega}\right)$, which is a contradiction.

Later in this section, we will show that this theorem is specific to $2^{\omega}$; it is not true when $2^{\omega}$ is changed to $3^{\omega}$. In particular, we will show that the equivalence relation of many-one equivalence on $3^{\omega}$ is a uniformly universal countable Borel equivalence relation.

We also remark that the statement of the theorem can be strengthened slightly:

Remark 3.14. The proof of Theorem 3.13 yields a statement that is actually slightly stronger than what we have stated. Because there is a uniform embedding of $F\left(\mathbb{F}_{\omega}, 2^{\omega}\right)$ into $F\left(\mathbb{F}_{2}, 2^{\omega}\right)$, the countable uniform joins that were used in this proof are very simple: they are computable compositions of two pairs of elements of $\left\{\varphi_{i}\right\}$. Hence Theorem 3.13 remains true when we assume closure under this smaller class of countable uniform joins.

It is interesting that our proof of Theorem 3.13 only uses that fact that the universal treeable equivalence relation can not be uniformly reduced to $E_{\left\{\varphi_{i}\right\}}$. Very little is known about what countable Borel equivalence relations 
can be reduced to Turing equivalence, or any other such $E_{\left\{\varphi_{i}\right\}}$ satisfying the hypothesis of Theorem 3.13. We make the following conjecture:

Conjecture 3.15. The universal treeable countable Borel equivalence relation is not Borel reducible to Turing equivalence.

Next, we will turn specifically to the case of equivalence relations coarser than Turing equivalence. We will begin by considering the following examples:

Definition 3.16. Let $\alpha$ be an ordinal less than $\omega_{1}^{c k}$ that is additively indecomposable so that if $\beta_{0}, \beta_{1}<\alpha$ then $\beta_{0}+\beta_{1}<\alpha$. Define $(<\alpha)$ reducibility, noted $\leq_{(<\alpha)}$, by $x \leq_{(<\alpha)} y$ if and only if there exists a $\beta<\alpha$ such that $x \leq_{T} y^{(\beta)}$ where $y^{(\beta)}$ is the $\beta$ th iterate of the Turing jump relative to $y$. (Our assumption here that $\alpha$ is additively indecomposable is needed so that $\leq_{(<\alpha)}$ is transitive). The symmetrization of this reducibility is the equivalence relation $\equiv_{(<\alpha)}$.

Hence, $\leq_{(<1)}$ is Turing reducibility, arithmetic reducibility is $\leq_{(<\omega)}$, and so on. Now $(<\alpha)$-reducibility is naturally generated by the functions obtained by taking the Turing reductions and the functions $x \mapsto x^{(\beta)}$ for all $\beta<\alpha$, and closing under composition. We assume henceforth that $(<\alpha)$ equivalence is generated by these canonical functions.

Note that we can relativize this definition to any $x \in 2^{\omega}$, and every additively indecomposable $\alpha<\omega_{1}^{x}$. Of course, different $x$ yield different equivalence relations, but any two such definitions of $(<\alpha)$-equivalence relations for the same ordinal $\alpha$ will agree on a Turing cone.

There is a certain sense in which $(<\alpha)$-reducibilities are the only "natural" computability-theoretic reducibilities coarser than $\leq_{T}$. We may justify this the following way. Suppose that $\leq_{P}$ is any countable Borel quasiorder that is coarser than Turing equivalence and closed under finite computable joins, i.e. if $x \geq_{P} y, z$, then $x \geq_{P} y \oplus z$. Then Slaman [37] has shown that on a Turing cone, $\leq_{P}$ is $(<\alpha)$-reducibility relative to $x$ for some $x$ and $\alpha<\omega_{1}^{x}$. Thus, up to a Turing cone, these reducibilities are the only ones coarser than Turing reducibility that are closed under finite computable joins.

We give a complete description of which of these equivalence relations are uniformly universal.

Theorem 3.17. Suppose $\alpha<\omega_{1}^{c k}$ is additively indecomposable. Then $(<$ $\alpha)$-equivalence is uniformly universal if and only if there is a $\beta<\alpha$ such that $\alpha=\beta \cdot \omega$. 
We prove the non-uniform universality here by using Remark 3.14. In the other case when $\alpha=\beta \cdot \omega$ for some $\beta<\alpha$, the proof of universality is an easy extension of Slaman and Steel's proof that arithmetic equivalence is universal. Recall from [30] that given $x: \omega \rightarrow 2^{<\omega}$ and $y \in 2^{\omega}$, we define $J(x, y) \in 2^{\omega}$ to be the real whose $n$th column is

$$
(J(x, y))^{[n]}= \begin{cases}x(n) 10000 \ldots & \text { if } \mathrm{y}(\mathrm{n})=0 \\ x(n) 01111 \ldots & \text { if } \mathrm{y}(\mathrm{n})=1\end{cases}
$$

We now give an extension of this definition that iterates this type of jump coding through the transfinite.

Definition 3.18. Given $x: \omega \rightarrow 2^{<\omega}$ and $y \in 2^{\omega}$, and a notation for $\alpha<$ $\omega_{1}^{c k}$, we define $J_{\alpha}(x, y) \in 2^{\omega}$ as follows. For $\alpha=1, J_{1}(x, y)=J(x, y)$. For $\alpha=\beta+1$ for $\beta>0$, we define $J_{\alpha}(x, y)=J\left(x_{0}, J_{\beta}\left(x_{1}, y\right)\right)$, where $x=x_{0} \oplus x_{1}$. Finally, suppose now that $\alpha$ is a limit ordinal and $\left(\lambda_{n}\right)_{n<\omega}$ is an computable sequence of computable ordinals whose limit is the computable ordinal $\alpha$. Then for $n \in \omega$, define the functions $f_{n}\left(x, y,\left(\lambda_{n}\right)\right)$ by

$$
f_{n}\left(x, y,\left(\lambda_{n}\right)\right)=J_{\lambda_{n}}\left(x^{[n]}, y(n)^{\frown} f_{n+1}\left(x, y,\left(\lambda_{n}\right)\right)\right)
$$

where $x^{[n]}$ is the $n$th column of $x$. Note that this definition is really an inductive definition of the $k$ th bit $f_{n}\left(x, y,\left(\lambda_{n}\right)\right)(k)$ simultaneously for all $n$; the definition of $f_{n}\left(x, y,\left(\lambda_{n}\right)\right)(k)$ will only use the values of $f_{n+1}\left(x, y,\left(\lambda_{n}\right)\right)\left(k^{\prime}\right)$ for $k^{\prime}<k$. Finally, define $J_{\alpha}(x, y)=f_{0}\left(x, y,\left(\lambda_{n}\right)\right)$.

Now we have the following:

Lemma 3.19. If $x_{0}, \ldots, x_{i}, z_{0}, \ldots, z_{j}$, and $w$ are mutually $\Delta_{1}^{1}$ generic functions from $\omega$ to $2^{<\omega}$, then for all $\alpha, \beta, \gamma<\omega_{1}^{\text {ck }}$ and $y_{0}, \ldots, y_{i} \in 2^{\omega}$

$$
\begin{aligned}
& \text { 1. }\left(0^{(\alpha)} \oplus J_{\beta}\left(x_{0}, y_{0}\right) \oplus \ldots \oplus J_{\beta}\left(x_{i}, y_{i}\right) \oplus z_{0} \oplus \ldots \oplus z_{j}\right)^{(\beta)} \\
& \qquad \equiv_{T} 0^{(\alpha+\beta)} \oplus x_{0} \oplus \ldots \oplus x_{i} \oplus y_{0} \oplus \ldots \oplus y_{i} \oplus z_{0} \oplus \ldots z_{j} \\
& \text { 2. } 0^{(\alpha)} \oplus J_{\beta}\left(x_{0}, y_{0}\right) \oplus \ldots \oplus J_{\beta}\left(x_{i}, y_{i}\right) \oplus z_{0} \oplus \ldots \oplus z_{j} \nsupseteq_{T} w
\end{aligned}
$$

Proof. This is an easy extension of the proof of [30, Lemma 2.4]. The successor step is essentially identical, and at limits we use the fact that the equivalences at each step are proved uniformly in $\beta$. 
Proof of Theorem 3.17. Suppose $\alpha<\omega_{1}^{c k}$ is closed under addition and there exists a $\beta<\alpha$ such that $\alpha=\beta \cdot \omega$. We must show that $\equiv_{(<\alpha)}$ is a universal countable Borel equivalence relation. It is enough to show that if $\mathbb{F}_{2}=\langle a, b\rangle$ acts on a Polish space $X$, then the resulting equivalence relation $E_{\mathbb{F}_{2}}^{X} \leq_{B} \equiv_{(<\alpha)}$. Let $g: X \rightarrow\left(2^{<\omega}\right)^{\omega}$ be a Borel function such that for every distinct $x_{0}, \ldots, x_{n} \in X$, we have that $g\left(x_{0}\right), \ldots, g\left(x_{n}\right)$ are all mutually $\Delta_{1}^{1}$ generic functions from $\omega$ to $2^{<\omega}$. The definition of the Borel reduction witnessing $E_{\mathbb{F}_{2}}^{X} \leq_{B} \equiv_{\alpha}$ is:

$$
f(x)=J_{\beta}\left(g(x), f(a \cdot x) \oplus f\left(a^{-1} \cdot x\right) \oplus f(b \cdot x) \oplus f\left(b^{-1} \cdot x\right)\right)
$$

Like Definition 3.18, this is really an inductive definition of $f(x)(k)$, which depends on values of $f(\gamma \cdot x)\left(k^{\prime}\right)$ for $\gamma \in\left\{a, a^{-1}, b, b^{-1}\right\}$, but only for $k^{\prime}<k$.

By inductively applying Lemma 3.19, we see that

$$
f^{(\beta \cdot n)}(x)=0^{(\beta \cdot n)} \oplus \bigoplus_{\left\{\gamma \in \mathbb{F}_{2}:|\gamma|<n\right\}} g(\gamma \cdot x) \oplus \bigoplus_{\left\{\gamma \in \mathbb{F}_{2}:|\gamma|=n\right\}} f(\gamma \cdot x)
$$

where $|\gamma|$ is the length of $\gamma$ as a word in $\mathbb{F}_{2}$. Hence, by part 2 of Lemma 3.19, we see that $f^{(\beta \cdot n)}(x)$ can not compute $g(y)$ for any $y$ not in the same $E_{\mathbb{F}_{2}}^{X}$ class of $x$. Thus, $f$ is an embedding, since therefore $f^{(\beta \cdot(n+1))}(x)$ can not compute $f(y)$ for all $n$ and all $y$ not in the same $E_{\mathbb{F}_{2}}^{X}$-class as $x$.

Now conversely, suppose that for every $\beta<\alpha$, we have that $\beta \cdot \omega<\alpha$. Now if we have pair of $(<\alpha)$-reductions, then they must both be $(<\beta)$ reductions for some $\beta<\alpha$. But then given any $x$, if we have a countable uniform of reals obtained by computably composing these two reductions applied to $x$, then it is still a $(<\alpha)$-reduction since $\beta \cdot \omega<\alpha$. Hence, by Remark 3.14, $(<\alpha)$-equivalence is not uniformly universal.

This proves part (4) of Theorem 1.6. This also proves part (3) of Theorem 1.5 since every countable Borel equivalence relation is contained in $(<\alpha)$-equivalence relative to some real. Note that the equivalence relations $\equiv_{(<\omega)}, \equiv_{\left(<\omega^{2}\right)}, \equiv_{\left(<\omega^{3}\right)}, \ldots$ are each uniformly universal by Theorem 3.17, but their union $\equiv_{\left(<\omega^{\omega}\right)}$ is not. This finishes the proof of part (4) of Theorem 1.5 together with Proposition 3.6.

\subsection{Contrasting results in the measure context and on $3^{\omega}$}

In this section, we shall prove a number of contrasting results to those in the previous section by showing that some of the equivalence relations considered 
there are universal if we change the space from $2^{\omega}$ to $3^{\omega}$, or are willing to discard nullsets. We will begin with some combinatorial results in the measure context which we will use in one of our constructions.

Lemma 3.20. Suppose $E$ and $F$ are countable Borel equivalence relations on a standard probability space $(X, \mu)$. Then there is a Borel set $A \subseteq X$ that meets $\mu$-a.e. E-class of cardinality $\geq 3$, and whose complement meets $\mu$-a.e. F-class of cardinality $\geq 3$.

Proof. We may find some standard Borel space $Y \supseteq X$ and equivalence relations $E^{*}$ and $F^{*}$ extending $E$ and $F$ such that all the $E^{*}$-classes and $F^{*}$ classes have cardinality $\geq 3$ and for all $x \in X$ whenever $[x]_{E}$ has cardinality $\geq 3$, then $[x]_{E}=[x]_{E^{*}}$ and when $[x]_{F}$ has cardinality $\geq 3$, then $[x]_{F}=[x]_{F^{*}}$. Now by [28, Theorem 1.7] there is a Borel set $A \subseteq Y$ such that $A$ meets $\mu$-a.e. $E^{*}$-class and the complement of $A$ meets $\mu$-a.e. $F^{*}$-class.

From this, we can conclude the following, which shows that Theorem 3.9 does not hold after discarding a nullset.

Lemma 3.21. Suppose that $X$ is a standard Borel space, $\left\{g_{i}\right\}_{i \in \omega}$ is a countable collection of partial Borel injections $X \rightarrow X$, and $\mu$ is a Borel probability measure on $X$. Then there is a Borel set $A$ of full measure and countably many Borel functions $c_{i}: A \rightarrow 2^{\omega}$ such that for all $x \in 2^{\omega}$, if $g_{i}(x)$ is defined and not equal to $x$ for all $i$, then there exists an $i$ such that $c_{i}(x) \neq c_{i}\left(g_{i}(x)\right)$.

Proof. We may assume all the $g_{i}$ are total Borel automorphisms of $X$. This is because we may extend the $g_{i}$ to total Borel automorphisms on some larger standard Borel space $Y \supseteq X$.

For each $i \in \omega$, let $E_{i}$ be the equivalence relation generated by $g_{i}$ and let $G_{i}$ be the graph generated by $g_{i}$.

We may prove this fact with merely two functions $c_{0}$ and $c_{1}$. By Lemma [3.20, there is a Borel set $B$ that meets $\mu$-a.e. $E_{0}$ class of cardinality $\geq 3$ and whose complement meets $\mu$-a.e. $E_{1}$ class of cardinality $\geq 3$. Let $G_{0}^{*}$ be the graph where we remove the edge between $x$ and $g_{0}(x)$ for every $x \in B$, and let $G_{1}^{*}$ be the graph where we remove the edge between $x$ and $g_{1}(x)$ for every $x \in B^{\mathrm{c}}$. Now there is a Borel set $A$ of full measure such that every connected component of $G_{0}^{*} \uparrow A$ and $G_{1}^{*} \uparrow A$ are finite. Finish by letting $c_{0}$ be a Borel 2-coloring of $G_{0}^{*} \uparrow A$ and $c_{1}$ be a Borel 2-coloring of $G_{1}^{*} \uparrow A$.

We are now ready to prove the following theorem: 
Theorem 3.22. Suppose $Z$ is some countable set (which is possibly empty) and $G$ is a countable group of permutations of the set $\mathbb{F}_{2} \times \omega \sqcup Z$ so that for every $\delta \in \mathbb{F}_{2}$, there exists some $\rho_{\delta} \in G$ so that $\rho_{\delta}((\gamma, n))=(\delta \gamma, n)$ for every $(\gamma, n) \in \mathbb{F}_{2} \times \omega$. Then

1. The permutation action of $G$ on $2^{\mathbb{F}_{2} \times \omega}$ generates a measure universal countable Borel equivalence relation.

2. The permutation action of $G$ on $3^{\mathbb{F}_{2} \times \omega}$ generates a universal countable Borel equivalence relation.

Suppose briefly that $Z$ is empty and we identify $2^{\mathbb{F}_{2} \times \omega}$ with $\left(2^{\omega}\right)^{\mathbb{F}_{2}}$. Then note that the permutations $(\gamma, n) \mapsto(\delta \gamma, n)$ which are required to be in the group $G$ generate the shift action of $\mathbb{F}_{2}$ on $\left(Y^{\omega}\right)^{\mathbb{F}_{2}}$; this is their significance.

Proof. Throughout we will let $Y \in\{2,3\}$. Let $E_{\infty}$ be a universal countable Borel equivalence relation generated by an action of $\mathbb{F}_{2}$ on a standard Borel space $X$. If $f: X \rightarrow Y^{\omega}$ is a function, then define the function $\hat{f}: X \rightarrow$ $Y^{\mathbb{F}_{2} \times \omega \sqcup Z}$ by

$$
\hat{f}(x)((\gamma, n))=f\left(\gamma^{-1} \cdot x\right)(n)
$$

for $(\gamma, n) \in F_{2} \times \omega$, and $\hat{f}(x)(z)=0$ for all $z \in Z$.

Note that if $x, y \in X$ and $\delta \cdot x=y$, then $\rho_{\delta} \cdot \hat{f}(x)=\hat{f}(y)$, since

$$
\begin{aligned}
\rho_{\delta} \cdot \hat{f}(x)((\gamma, n))=\hat{f}(x) & \left(\rho_{\delta}^{-1}((\gamma, n))\right)=\hat{f}(x)\left(\left(\delta^{-1} \gamma, n\right)\right) \\
& =f\left(\gamma^{-1} \cdot \delta \cdot x\right)(n)=\hat{f}(\delta \cdot x)((\gamma, n))=\hat{f}(y)((\gamma, n))
\end{aligned}
$$

So given any Borel $f$, the associated $\hat{f}$ is a Borel homomorphism from $E_{\infty}$ to the orbit equivalence relation of the permutation action of $G$ on $Y^{\mathbb{F}_{2} \times \omega \sqcup Z}$. We will define a Borel function $f$ so that the corresponding $\hat{f}$ becomes our desired Borel reduction.

Say a permutation $\rho \in G$ uses $\{n, m\}$ for $n \neq m \in \omega$ if there exist group elements $\delta, \gamma \in \mathbb{F}_{2}$ such that $\rho((\gamma, n))=(\delta, m)$. Let $G^{\prime}$ be the set of $\rho \in G^{\prime}$ so $\rho$ does not use infinitely many pairs $\{n, m\}$, and there are only finitely many $n$ such that $\rho(Z) \cup \rho^{-1}(Z)$ meets $\mathbb{F}_{2} \times\{n\}$. Note that $G^{\prime}$ is a subgroup of $G$.

Let $S_{0}, S_{1} \subseteq \omega$ be disjoint sets so that $S_{0} \cup S_{1}$ is coinfinite, and

1. For every $\rho \in G$ that uses infinitely many pairs $\{n, m\}$, there is some pair $\{n, m\}$ used by $\rho$ so that $n \in S_{0}$ and $m \in S_{1}$.

2. For every $\rho \in G$ such that there are infinitely many $n$ such that $\rho(Z) \cup$ $\rho^{-1}(Z)$ meets $F_{2} \times\{n\}, \rho(Z) \cup \rho^{-1}(Z)$ meets $\mathbb{F}_{2} \times S_{1}$. 
Our first constraint on the function $f$ will be that for every $x \in X$,

$$
f(x)(n)=0 \wedge f(x)(m)=1 \text { for every } n \in S_{0} \text { and } m \in S_{1} .
$$

We claim now that if $\rho \in G$, but $\rho \notin G^{\prime}$, then $y \in \operatorname{ran}(\hat{f})$ will imply that $\rho \cdot y \notin \operatorname{ran}(\hat{f})$. This is because if $\rho((\gamma, m))=(\delta, n)$ and $n \in S_{0}$ and $m \in S_{1}$, then for all $y \in \operatorname{ran}(\hat{f})$, we have $y((\gamma, m))=1$ and $y((\delta, n))=0$. However, $(\rho \cdot y)((\delta, n))=y((\gamma, m))=1$, and so $(\rho \cdot y) \notin \operatorname{ran}(\hat{f})$. We argue similarly for $\rho$ such that $\rho(Z) \cup \rho\left(Z^{-1}\right)$ meets $\mathbb{F}_{2} \times S_{1}$.

Let $S_{2} \subseteq \omega$ be an infinite set disjoint from $S_{0} \cup S_{1}$ and so that $S_{0} \cup S_{1} \cup S_{2}$ is coinfinite. Let $h: X \rightarrow 2^{S_{2}}$ be a Borel reduction from the equality relation on $X$ to the relation of equality mod finite on $2^{S_{2}}$. (For example, we may assume $X=2^{\omega}$, let $\pi: S_{2} \rightarrow \omega$ be an infinite-to-one surjection, and let $h(x)(n)=x(\pi(n))$.$) Now define$

$$
f(x)(n)=h(x)(n) \text { for every } n \in S_{2}
$$

Suppose $\rho \in G^{\prime}$. Then for sufficiently large $n \in S_{2}$, we must have that for every $x \in X,(\rho \cdot \hat{f})(x)(n)$ has been defined by $\left(^{*}\right)$. Because we can recover $y \in X$ if we know all but finitely many bits of $h(y)$, there can be at most one $y$ such that for all sufficiently large $n \in S_{2}$, we have $(\rho \cdot \hat{f})(x)(\gamma, n)=\hat{f}(y)(\gamma, n)$ for all $\gamma \in \mathbb{F}_{2}$. Let $g_{\rho}: X \rightarrow X$ be the partial Borel function mapping each $x \in X$ to this unique $y \in X$ if it exists. Note that if $g_{\rho}(x)=y$ then $g_{\rho^{-1}}(y)=x$, so $g_{\rho}$ is a partial injection. For each $\rho$, also define $n_{\rho}$ to be least such that for every $n \geq n_{\rho}$ and $\gamma \in F_{2}$ there is a $\delta \in \mathbb{F}_{2}$ such that $\rho((\gamma, n))=(\delta, n)$.

For each $n \geq n_{\rho}$, let $g_{\rho, n}: X \rightarrow X$ be the partial Borel function so $g_{\rho, n}(y)=\gamma \cdot g_{\rho}^{-1}(y)$ where $\gamma \in \mathbb{F}_{2}$ is such that $\rho^{-1}((1, n))=(\gamma, n)$. Hence, if $g_{\rho}(x)=y$, and $x E_{\infty} y$ then $\rho \cdot \hat{f}(x)=\hat{f}(y)$ would imply $f(y)(n)=$ $\hat{f}(y)((1, n))=(\rho \cdot \hat{f}(x))((1, n))=\hat{f}(x)\left(\rho^{-1}(1, n)\right)=\hat{f}(x)((\gamma, n))=f\left(\gamma^{-1}\right.$. $x)(n)=f\left(g_{\rho, n}(y)\right)(n)$ for every $n \geq n_{\rho}$.

Hence, to make $\hat{f}$ a Borel reduction, it suffices to ensure that for every $y \in X$ and every $\rho \in G^{\prime}$ if $g_{\rho}^{-1}(y)$ is defined, then either $g^{-1}(y) E_{\infty} y$, or else there is an $n \geq n_{\rho}$ such that $f(y)(n) \neq f\left(g_{\rho, n}(y)\right)$.

For each $\rho \in G^{\prime}$ let $S_{3, \rho}$ be an infinite set disjoint from $S_{0} \cup S_{1} \cup S_{2}$ with $\min \left(S_{3, \rho}\right) \geq n_{\rho}$, and so that the sets $\left\{S_{3, \rho}\right\}_{\rho \in G^{\prime}}$ are all pairwise disjoint. Define $f(x)(n)$ arbitrarily for $n \notin S_{0} \cup S_{1} \cup S_{2} \cup\left(\bigcup\left\{S_{3, \rho}\right\}_{\rho \in G^{\prime}}\right)$.

We will now indicate how to finish the construction to show both parts (1) and (2).

To prove (1), suppose $\mu$ is a Borel probability measure on $X$. By Lemma 3.21, for each $\rho \in G^{\prime}$ and $n \in S_{3, \rho}$, let $A_{\rho}$ be a $\mu$-conull Borel set 
and let $c_{\rho, n}: A_{\rho} \rightarrow 2$ be a function so that for every $y \in A_{\rho}$, there is some $n \in S_{3, \rho}$ so that $g_{\rho, n}(y)=y, g_{\rho, n}(y)$ is undefined, or $c_{\rho, n}(y) \neq c_{\rho, n}\left(g_{\rho, n}(y)\right)$. We finish our definition of $f$ by letting

$$
f(y)(n)=c_{\rho, n}(y) \text { for all } \rho \in G^{\prime} \text { and } n \in S_{3, \rho}
$$

We claim that $\hat{f} \uparrow \bigcap_{\rho \in G^{\prime}} A_{\rho}$ a reduction. Above, we have already shown that if $\rho \notin G^{\prime}$, then $\rho \cdot \hat{f}(y)$ is not in the range of $\hat{f}$ for all $y \in X$. So suppose $\rho \in G^{\prime}$ and $y \in A_{\rho}$. Then if $g_{\rho}^{-1}(y)$ is defined and $g_{\rho}^{-1}(y) E E_{\infty} y$, this implies that $g_{\rho, n}(y) \neq y$ for all $n$, hence there is some $n \in S_{3, \rho}$ such that $c_{\rho, n}(y) \neq c_{\rho, n}\left(g_{\rho, n}(y)\right)$. Hence, $\rho^{-1} \cdot \hat{f}(y)$ is not in the range of $\hat{f}$.

To prove (2), each injective partial function $g_{\rho, n}$ generates a Borel graph on $X$ of degree at most 2. Let $c_{\rho, n}: X \rightarrow 3$ be a Borel 3-coloring of this graph by [20, Proposition 4.6], and define

$$
f(y)(n)=c_{\rho, n}(y) \text { for all } \rho \in G^{\prime} \text { and } n \in S_{3, \rho} .
$$

Recall that given $x, y \in 2^{\omega}$, we say that $x$ is many-one reducible to $y$, noted $x \leq_{m} y$, if there is a computable function $r: \omega \rightarrow \omega$ such that $x=r^{-1}(y)$. The associated symmetrization of this reducibility is many-one equivalence, and is noted $\equiv_{m}$. Many-one equivalence is Borel reducible to recursive isomorphism via the function $x \mapsto \oplus_{i \in \omega} x$; the function mapping $x$ to the computable join of $\omega$ many copies of $x$. Indeed, for all reals $x$ and $y$, we have that $\oplus_{i \in \omega} x$ and $\oplus_{i \in \omega} y$ are recursively isomorphic if and only if they are many-one equivalent. Note that many-one equivalence is closed under countable uniform joins.

\section{Corollary 3.23.}

1. The equivalence relation of recursive isomorphism on $2^{\omega}$ is measure universal.

2. The equivalence relation of recursive isomorphism on $3^{\omega}$ is universal.

Further, the same two facts are true for many-one equivalence in place of recursive isomorphism.

Proof. Take a computable bijection between $\mathbb{F}_{2} \times \omega$ with $\omega$ so we can identify $2^{\omega}$ with $2^{\mathbb{F}_{2} \times \omega}$, and similarly for the base space 3 . Then the group of computable permutations of $\omega$ includes the permutations required in the assumptions of Theorem 3.22 . 
The fact that these facts are also true for many-one equivalence follows from the above observation that we may change the group $\mathbb{F}_{2}$ in the proof of Theorem 3.22 into the group $\mathbb{F}_{3}$, and the proof will work unchanged to construct a Borel reduction from the equivalence relation generated by any Borel action of $\mathbb{F}_{3}$ on $X$. Now take an action of $\mathbb{F}_{3}=\langle a, b, c\rangle$ where the first two generators $a, b$ generate a universal countable Borel equivalence relation, and the last generator $c$ acts trivially so that $c \cdot x=x$ for every $x \in X$. Then the range of the resulting $\hat{f}$ will have $\hat{f}(x)\left(\left(c^{k} \delta, n\right)\right)$ for every $k \in \mathbb{Z}$. Thus, every bit in $\hat{f}(x)$ is duplicated infinitely many times, and so $\hat{f}(x)$ and $\hat{f}(y)$ are many-one equivalent iff they are recursively isomorphic.

Now recursive isomorphism on $2^{\omega}$ is not closed under countable uniform joins, and hence Theorem 3.13 does not apply to it. However, because of the close connection between recursive isomorphism and many-one equivalence, and the fact that all known approaches to the universality of recursive isomorphism also give the universality of many-one equivalence, we make the following conjecture:

Conjecture 3.24. Recursive isomorphism on $2^{\omega}$ is not a universal countable Borel equivalence relation.

We can now prove part (2) of Theorem 1.5.

Theorem 3.25. Given any uniformly universal countable Borel equivalence relation $E_{\left\{\varphi_{i}\right\}}^{X}$ on a standard probability space $(X, \mu)$, there is an invariant Borel set $A$ of full measure such that $E_{\left\{\varphi_{i}\right\}}^{X} \uparrow A$ is not uniformly universal.

Proof. There is a uniform embedding of $E_{\left\{\varphi_{i}\right\}}^{X} \uparrow A$ into many-one equivalence for some $\mu$-conull Borel set $A$ by Corollary 3.23 . However, since many-one equivalence is not uniformly universal by Theorem $3.13, E_{\left\{\varphi_{i}\right\}}^{X} \uparrow A$ can not be uniformly universal.

Our proofs above give the following interesting consequence about treeable equivalence relations.

Theorem 3.26. Let $E_{\infty T}=F\left(\mathbb{F}_{2}, 2\right)$ be the universal treeable countable Borel equivalence relation, and let $\mu$ be a Borel probability measure on the free part of $2^{\mathbb{F}_{2}}$. Then there exists a $\mu$-conull Borel set $A$ such that $E_{\infty \mathcal{T}}$ does not uniformly reduce to $E_{\infty} \uparrow \uparrow$.

Proof. In the proof of Theorem [3.13, we see that $E_{\infty \mathcal{T}}$ does not uniformly reduce to many-one equivalence on $2^{\omega}$. However, we have shown that manyone equivalence is measure universal, and hence $E_{\infty \mathcal{T}} \uparrow A$ does uniformly 
reduce to recursive isomorphism on $2^{\omega}$ for some conull set $A$ with respect to every Borel probability measure. Hence, we see that $E_{\infty} \mathcal{T}$ cannot uniformly reduce to $E_{\infty \mathcal{T}} \uparrow A$.

Hence, it seems reasonable to expect that the Borel cardinality of $E_{\infty T}$ decreases after we discard a sufficiently complicated nullset, with respect to any Borel probability measure. At the very least, any proof that this is not the case must use a complicated nonuniform construction.

In more recent work joint with Jay Williams, we have generalized part (2) of Theorem 3.23 as follows.

Theorem 3.27 (joint with Jay Williams). Suppose $X$ is a Polish space with $|X| \geq 3$, and $G$ is any countable subgroup of $S_{\infty}$. Then the permutation action of $G$ on $X^{\omega}$ generates a uniformly universal countable Borel equivalence relation if and only if there exists some $k \in \omega$ and a subgroup $H \leq G$ isomorphic to $\mathbb{F}_{2}$ such that the map $H \rightarrow \omega$ given by $\rho \mapsto \rho(k)$ is injective.

Essentially, not only must $\Gamma$ contain a copy of $\mathbb{F}_{2}$, as given in (1) of Theorem 1.5, this must be witnessed in a single orbit.

Proof. For the forward direction, assume that for all $n \in \omega$ and $g_{0}, g_{1} \in G$, that there exists some nontrivial reduced word $h$ in $g_{0}$ and $g_{1}$ such that $h(n)=n$. Now let $f$ be a uniform Borel homomorphism from $E\left(\mathbb{F}_{2}, 2^{\omega}\right)$ to $E_{G}^{X^{\omega}}$ with uniformity function $u: \mathbb{F}_{2} \rightarrow G$. Since $\mathbb{F}_{2}$ is a free group, we may assume that $u$ is a group homomorphism. We claim that $f$ is constant on a set of Lebesgue measure 1. It is enough to show that for each $n \in \omega$, $f(x)(n)$ is constant on a set of Lebesgue measure 1 .

Let $k \in \omega$ be given. Let $\mathbb{F}_{2}=\langle\alpha, \beta\rangle$ and consider words in $u(\alpha)$ and $u(\beta)$. By assumption, there must be some nonidentity $\gamma \in \mathbb{F}_{2}$ such that $u(\gamma)(k)=k$. We see that $f(\gamma \cdot x)(k)=u(\gamma) \cdot f(x)(k)=f(x)\left(u(\gamma)^{-1}(k)\right)=$ $f(x)(k)$. Hence, the value assigned to $x$ by $x \mapsto f(x)(k)$ is invariant under the map $x \mapsto \gamma \cdot x$. However, since $x \mapsto \gamma \cdot x$ is an ergodic transformation, the map $x \mapsto f(x)(n)$ must therefore be constant a.e. (Note that here we have not used the fact that the cardinality of $X$ is $\geq 3$.)

The reverse direction follows from Lemma 3.22, By assumption there is a subgroup $H \leq G$ isomorphic to $\mathbb{F}_{2}$ and a $k \in \omega$ so that the map $H \mapsto \omega$ defined by $h \mapsto h(k)$ is injective. Let $H^{\prime} \leq H$ be a subgroup isomorphic to $\mathbb{F}_{3}$, and let $\alpha, \beta, \xi \in H^{\prime}$ be generators of this subgroup. Let $\phi: \mathbb{F}_{2} \rightarrow H^{\prime}$ be an isomorphism sending the two generators of $\mathbb{F}_{2}$ to $\alpha$ and $\beta$, and let $\pi: \mathbb{F}_{2} \times \omega \rightarrow \omega$ be the injection $\pi(\gamma, i)=\phi(\gamma) \circ \xi^{i}(k)$, so we can 
view $\omega$ as $\operatorname{ran}(\pi) \sqcup(\omega \backslash \operatorname{ran}(\pi))$, and canonically identify it with $\mathbb{F}_{2} \times \omega \sqcup Z$ for $Z=\omega \backslash \operatorname{ran}(\pi)$. Viewed this, way $G$ therefore contains the required permutations in order to apply Lemma 3.22 to show that the permutation action of $G$ on $3^{\omega}$ is universal.

Note that one implication of this theorem is that if $G \leq H \leq S_{\infty}$ are countable and the permutation action of $G$ on $X^{\omega}$ generates a uniformly universal countable Borel equivalence relation, then so does the permutation action of $H$.

With this theorem, we have finished proving all the abstract properties and classifications of uniform universality that we have discussed in the introduction. 


\section{Ultrafilters on quotient spaces}

\subsection{An introduction to ultrafilters on quotient spaces}

Largeness notions for the subsets of a standard Borel space play a central role in descriptive set theory. They are an indispensable tool whenever we want to prove an impossibility result such as $E \not_{B} F$ for some Borel equivalence relations $E$ and $F$. In such situations, we must analyze all possible Borel functions that could be counterexamples to these statements. While we have no hope of understanding the behavior of arbitrary Borel functions everywhere, if we are willing to discard some "small" part of the domain of the function, we may gain a much clearer understanding of its behavior on the remaining "large" complement.

Formally, a notion of largeness is often taken to mean a $\sigma$-complete filter, whose corresponding notion of smallness is its dual $\sigma$-ideal. For example, Baire category and Borel probability measures yield such notions of largeness: the comeager sets and conull sets. Our main goal in this section will be to define largeness notions that are particularly well suited for studying Borel equivalence relations (though one could equally well study other types of Borel objects, such as Borel graphs, or Borel quasiorders). They will have much stronger properties than merely being $\sigma$-complete filters.

As a motivating example, consider $E_{0}$ : the equivalence relation of equality mod finite on infinite binary sequences in $2^{\omega}$. Both Lebesgue measure and Baire category have two properties that are particularly helpful when they are used to analyze homomorphisms from $E_{0}$ to other Borel equivalence relations. First, every $E_{0}$-invariant Borel set $A \subseteq 2^{\omega}$ has either Lebesgue measure 0 or 1 , and likewise is either meager or comeager. Hence, with respect to either of these two notions, every $E_{0}$-invariant set is either large or small. Phrased another way, these largeness notions are ultrafilters when restricted to $E_{0}$-invariant sets. Second, since the restriction of $E_{0}$ to a comeager or conull set cannot be smooth by standard ergodicity arguments, it must therefore be Borel bireducible with $E_{0}$ by the Glimm-Effros dichotomy [13].

We isolate these two phenomena into the following definitions. Recall that given any $\sigma$-algebra $\Sigma$, an ultrafilter on $\Sigma$ is a maximal filter of $\Sigma$. An ultrafilter $U$ is said to be $\sigma$-complete if the intersection of countably many elements of $U$ is in $U$.

Definition 4.1. Suppose $E$ is a countable Borel equivalence relation on a standard Borel space $X$ with the associated $\sigma$-algebra $\Sigma$ of $E$-invariant Borel sets. A $\sigma$-complete ultrafilter on the Borel subsets of $X / E$ is a $\sigma$ - 
complete ultrafilter $U$ on $\Sigma$. $U$ is said to preserve the Borel cardinality of $E$ if for all $A \in U$, we have $E \sim_{B} E \uparrow A$.

Let us expand our horizons briefly and discuss this situation under the axiom of determinacy. Under $\mathrm{AD}$, every ultrafilter must be $\sigma$-complete (see for instance [18, Proposition 28.1]) and so there can not be any nonprincipal ultrafilter on any Polish space. Nevertheless, many ultrafilters exist on quotients of Polish spaces by equivalence relations. For example, the two examples of ultrafilters we have given above as well as all the examples we will give below will easily extend to define ultrafilters on all subsets of the quotient $X / E$ under $\mathrm{AD}$. Hence, the reader may assume we are working in this context if they prefer, and work with genuine ultrafilters for the $\sigma$-algebra of all subsets of $X / E$, instead of just the Borel ones.

We mention here that there are several open questions concerning the structure of the ultrafilters on the quotient space of a Borel equivalence relation $E$. Abstractly, we would like to know if there is a way of classifying such ultrafilters and hence understanding exactly what types of tools exist with which we can analyze $E$ using ergodicity arguments. The most natural way of organizing these ultrafilters is by Rudin-Keisler reducibility. Several important open questions can be rephrased in this framework. For example, the question of whether Martin's ultrafilter is $E_{0}$-ergodic [40] is equivalent to asking whether there is a nonprincipal ultrafilter on the quotient of $E_{0}$ that is Rudin-Keisler reducible to Martin's ultrafilter on the Turing degrees. Along these lines, Zapletal has also asked whether the ultrafilters arising from measure and category are a basis for all the ultrafilters on the quotient of $E_{0}$.

As described in the introduction, our main goal in this section is the construction of new ultrafilters on the quotient space of countable Borel equivalence relations that do not arise from measure or category. We are motivated to construct ultrafilters in particular because of the central role that ergodicity plays in the subject, and because of connections with Martin's ultrafilter on the Turing degrees. Further, our desire for ultrafilters which preserve Borel cardinality comes from our ultimate goal of proving sharper theorems than those which are currently possible using category (which suffers from generic hyperfiniteness [24, Theorem 12.1]), and measure (which we conjecture does not preserve Borel cardinality in many natural cases such as for $E_{\infty T}$ and $E_{\infty}$ : see Theorems 3.25 and 3.26).

The search for tools beyond measure theory to use in the study of countable Borel equivalence relations has long been a central question of the subject. Indeed, up to the present, in every case where we can prove that 
there is no definable reduction from $E$ to $F$ for countable Borel equivalence relations $E$ and $F$, we have been able to give a measure-theoretic proof of this fact. We may make this question precise under the axiom $\mathrm{AD}^{+}$, a slight technical strengthening of AD due to Woodin which implies uniformization for subsets of $\mathbb{R}^{2}$ with countable sections [45] 4. Assume $\mathrm{AD}^{+}$, and suppose $E$ and $F$ are countable equivalence relations on the standard Borel space $X$ and $Y$. Then say $E$ is reducible to $F$ if there is a function $f: X \rightarrow Y$ such that $x E y \Longleftrightarrow f(x) F f(y)$. Say that $E$ is measure reducible to $F$ if for every probability measure $\mu$ on $X$, there exists a $\mu$-conull set $A$ such that $E \uparrow A$ is reducible to $F$. It is open whether these two notions are distinct. That is, whether every instance of nonreducibility arises for measure-theoretic reasons.

Question $4.2\left(\mathrm{AD}^{+}\right)$. If $E$ and $F$ are countable equivalence relations on standard Borel spaces and $E$ is measure reducible to $F$, then must $E$ be reducible to $F$ ?

An affirmative answer to Question 4.2 seems unlikely. For example, we have shown above that $E_{\infty}$ is measure reducible to the equivalence relation of recursive isomorphism on $2^{\omega}$ and also conjectured that recursive isomorphism on $2^{\omega}$ is not universal (see Theorem 3.23 and Conjecture 3.24).

We return now to the realm of Borel sets. The existence of a $\sigma$-complete ultrafilter on the Borel subsets of $X / E$ already yields some interesting information about $E$, related to its indivisibility, which we proceed to define:

Definition 4.3. Suppose that $E$ and $F$ are Borel equivalence relations. Say that $E$ is $F$-indivisible if for every Borel homomorphism $f$ from $E$ to $F$, there exists an $F$ equivalence class $C$ such that $E \uparrow f^{-1}(C)$ is Borel bireducible with $E$. In the case where $F$ is the relation of equality on a standard Borel space of cardinality $\kappa$, then we say that $E$ is $\kappa$-indivisible.

Given Borel equivalence relations $E$ and $F, E$ being $F$-indivisible is perhaps the antithesis of having $E$ Borel reducible to $F$. Not only is $E \not_{B} F$, but any homomorphism $f$ from $E$ to $F$ makes no progress whatsoever in completely classifying $E$, since there is a single $F$-class whose preimage under $f$ has the same Borel cardinality as $E$.

We know many examples of interesting pairs of uncountable Borel equivalence relations $E$ and $F$ for which $E$ is $F$-indivisible (see for example

\footnotetext{
${ }^{4}$ The Borel version of Question 4.2 is false because of the known difference between $\sigma$ $\Sigma_{1}^{1}$ reducibility and Borel reducibility for countable Borel equivalence relations [1, Theorem $5.5]$
} 
the book of Kanovei, Sabok, and Zapletal [19]). However, no countable Borel equivalence relations beyond $E_{0}$ were known even to be indivisible into two pieces until we showed this for $E_{\infty}$ in [30]. In contrast, we note that Thomas [39] has shown the existence of a countable Borel equivalence relation $E$ on an uncountable standard Borel space that is not 2-indivisible. Indeed, there is a countable Borel equivalence relation $E$ where $E<_{B} E \oplus E$, where $E \oplus E$ is the direct sum of two disjoint copies of $E$.

Our study of ultrafilters is connected to indivisibility in the following way:

Proposition 4.4. Suppose there exists a $\sigma$-complete Borel cardinality preserving ultrafilter $U$ on the Borel E-invariant sets. Then $E$ is $2^{\aleph_{0}}$-indivisible.

Proof. This is a standard ergodicity argument. Let $f$ be a Borel homomorphism from $E$ to $\Delta\left(2^{\omega}\right)$. For each $n \in \omega$, let $A_{n, 0}=\{x: f(x)(n)=0\}$ and $A_{n, 1}=\{x: f(x)(n)=1\}$. Then for each $n$, either $A_{n, 0} \in U$ or $A_{n, 1} \in U$. Hence, there is an $x \in 2^{\omega}$ such that $A_{n, x(n)} \in U$ for all $n$ and so $A=f^{-1}(x)=\cap_{n} A_{n, x(n)}$ has $A \in U$, so $E \uparrow A \sim_{B} E$.

In [30] we pointed out that for arithmetic equivalence, the invariant sets containing an arithmetic cone are a $\sigma$-complete ultrafilter on the $\equiv_{A^{-}}$ invariant sets. Since Slaman and Steel have shown that arithmetic equivalence is a universal countable Borel equivalence relation, and their proof relativizes, we noted that this implies that this ultrafilter preserves Borel cardinality and hence that $E_{\infty}$ is $2^{\aleph_{0}}$-indivisible. This answered questions of Jackson, Kechris and Louveau, [17] and Thomas [41, question 3.20], who had asked whether $E_{\infty}$ is 2-indivisible and $2^{\aleph_{0}}$-indivisible respectively. In this section, we will give a new proof of this result by constructing a large family of cardinality preserving ultrafilters on the quotient of countable Borel equivalence relations of which $E_{\infty}$ is merely one example.

It remains an open question to classify exactly which countable Borel equivalence relation $E$ are such that $E_{\infty}$ is $E$-indivisible. However, Martin's conjecture provides an answer to this question, as discussed in [30].

\subsection{A natural ultrafilter on the quotient of $E\left(\mathbb{F}_{\infty}, 2^{\omega}\right)$}

As in Section 2, throughout this section we will have $I \leq \omega$ and $\left\{\Gamma_{i}\right\}_{i \in I}$ a collection of disjoint countable groups. For each $i \in I$, we also fix a listing $\gamma_{i, 0}, \gamma_{i, 1} \ldots$ of the nonidentity elements of $\Gamma_{i}$.

We will begin by proving a strengthening of Lemma 2.8 to show that $f$ may be chosen to be a Borel reduction. This will require us to generalize slightly the main game from Section 2 . 
Definition 4.5 (The general game). Fix a strictly increasing sequence of natural numbers $\left(n_{k}\right)_{k \in \omega}$. We define the general game $G_{i}^{A}\left(\left(n_{k}\right)\right)$ for producing $y \in\left(\prod_{i} 2^{\omega}\right)^{*_{i} \Gamma_{i}}$ identically to the game $G_{i}^{A}$, except with the following modification: the appropriate player will define $y(\alpha)(i)(n)$ on turn $k$ of the game if $t(\alpha) \geq k$, and $n_{(k-t(\alpha))} \leq\langle i, n\rangle<n_{(k+1-t(\alpha))}$.

Hence, the main game $G_{i}^{A}$ from Definition 2.4 corresponds to the general game $G_{i}^{A}\left(\left(n_{k}\right)\right)$ for the sequence $n_{k}=k$. It is easy to check that for all the lemmas in Sections 2.1 and 2.2 that use game $G_{i}^{A}$, we can use the more general game $G_{i}^{A}\left(\left(n_{k}\right)\right)$ instead. In all the relevant inductions where we assume the turns $<k$ have been played, one simply replaces the assumption that $y(\alpha)(i)(n)$ has been defined for $t(\alpha)+\langle i, n\rangle<k$ with the assumption that $y(\alpha)(i)(n)$ has been defined where $t(\alpha)<k$ and $\langle i, n\rangle<n_{k-t(\alpha)}$.

We have one more technical definition giving a growth criterion for sequences $\left(n_{k}\right)_{k \in \omega}$.

Definition 4.6. Let $S_{k}=\left\{\beta \in \Gamma_{j}: t(\beta) \leq k\right\}$. Let $N_{\alpha, i, k}=\{n \in$ $\left.\omega: n_{k-t\left(\alpha^{-1}\right)} \leq\langle i, n\rangle \leq n_{k+1-t\left(\alpha^{-1}\right)}\right\}$, so that the $n$th bit of $(\alpha \cdot y)(1)(i)$ (which is equal to the $n$th bit of $y\left(\alpha^{-1}\right)(i)$ ) is determined on turn $k$ if and only if $n \in N_{\alpha, i, k}$. Say that a sequence $\left(n_{k}\right)_{k \in \omega}$ is good if for every $i \in I$, $d \in \omega$, and $\alpha \in *_{i} \Gamma_{i}$, there are infinitely many $k$ such that $d^{\left|S_{k}\right|}<2^{\left|N_{a, i, k}\right|}$.

Lemma 4.7. Assume that $\left\{A_{i}\right\}_{i \in I}$ is a Borel partition of $\left(\prod_{i} 2^{\omega}\right)^{*_{i} \Gamma_{i}}$ and that player II has a winning strategy in the game $G_{j}^{A_{j}}\left(\left(n_{k}\right)\right)$ for a good sequence $\left(n_{k}\right)_{k \in \omega}$. Then there exists some $j \in I$ and an injective continuous $\Gamma_{j}$-equivariant function $f:\left(2^{\omega}\right)^{\Gamma_{j}} \rightarrow A_{j}$, but with the additional property that $f$ is an embedding of $E\left(\Gamma_{j}, 2^{\omega}\right)$ into $E\left(*_{i} \Gamma_{i}, \prod_{i} 2^{\omega}\right)$.

Proof. Fix such a winning strategy. The $f$ we produce will be a slight variation on the one produced in Lemma 2.8. Given any $g:\left(2^{\omega}\right)^{\Gamma_{j}} \rightarrow 2^{\omega}$, let $f_{g}$ be the unique equivariant Borel function such that for all $x \in 2^{\omega}$ we have $f_{g}(x)(\gamma)(j)=g\left(\gamma^{-1} \cdot x\right)$, and such that $f_{g}(x)$ is a winning outcome of player II's winning strategy in the game $G_{j}^{A_{j}}\left(\left(n_{k}\right)_{k \in \omega}\right)$. This $f_{g}$ exists by an analogous argument to that in the proof of Lemma 2.8. In this notation, the $f$ produced in the proof of Lemma 2.8 is the function $f_{g}$ where $g(x)=x(1)$. We claim that if $g$ is a sufficiently generic continuous function, then $f_{g}$ will be as desired.

To simplify notation, identify $\left(2^{\omega}\right)^{\Gamma_{j}}$ with $2^{\Gamma_{j} \times \omega}$. Fix some enumeration of $\Gamma_{j} \times \omega$, and let $2^{<\Gamma_{j} \times \omega}$ be the set of all functions from some finite initial segment of $\Gamma_{j} \times \omega$ to 2 . Hence, if $s, t \in 2^{<\Gamma_{j} \times \omega}$ are compatible, then $s \subseteq t$ or $t \subseteq s$. In general, if $s, t \in 2^{\Gamma_{j} \times \omega}$ and $\beta \in \Gamma_{j}$, we will say that $\beta \cdot s$ is 
compatible with $t$ if for all $(\gamma, n) \in \Gamma_{j} \times \omega$ so that both $s\left(\left(\beta^{-1} \gamma, n\right)\right)$ and $t((\gamma, n))$ are defined, we have $s\left(\left(\beta^{-1} \gamma, n\right)\right)=t((\gamma, n))$. Otherwise, we will say that $\beta \cdot r$ is incompatible with $s$.

The sense of genericity that we mean for continuous functions from $\left(2^{\omega}\right)^{\Gamma_{j}} \rightarrow 2^{\omega}$ will be the usual one. Our set $\mathbb{P}$ of conditions will be the set of all finite partial functions $p$ from $2^{<\Gamma_{j} \times \omega} \rightarrow 2^{<\omega}$ such that if $s, t \in \operatorname{dom}(p)$ and $s \subseteq t$, then $p(s) \subseteq p(t)$. It is clear that a generic filter for this poset will yield a unique continuous function $g:\left(2^{\omega}\right)^{\Gamma_{j}} \rightarrow 2^{\omega}$ in the usual way.

Note that a sufficiently generic function $g:\left(2^{\omega}\right)^{\Gamma_{j}} \rightarrow 2^{\omega}$ will have the property that there exists a function $\rho_{j}: \omega \rightarrow \omega$ such that for all $x \in\left(2^{\omega}\right)^{\Gamma_{j}}$, $\gamma \in \Gamma_{j}$ and $n \in \omega$, we have $x(1)(n)=g(x)\left(\rho_{j}(n)\right)$. Hence, $g$ will be injective, and thus so will $f_{g}$.

We must show that whenever $x, y \in\left(2^{\omega}\right)^{\Gamma_{j}}$ are not $E\left(\Gamma_{j}, 2^{\omega}\right)$-related, and $\alpha \in *_{i} \Gamma_{i}$ is not an element of $\Gamma_{j}$, then $\alpha \cdot f_{g}(x) \neq f_{g}(y)$. Of course, if $\alpha \in \Gamma_{j}$, then $\alpha \cdot f_{g}(x)=f_{g}(\alpha \cdot x)$, since $f_{g}$ is $\Gamma_{j}$-equivariant.

Fix $\alpha \in *_{i} \Gamma_{i}$. It suffices to prove the following. Suppose $r, s \in 2^{<\Gamma_{j} \times \omega}$ are such that $\beta \cdot r$ is incompatible with $s$ for all $\beta \in S_{t(\alpha)}$. Then given any condition $p \in P$, it is dense to extend $p$ to a condition $p^{*}$ such that for all continuous functions $g$ extending $p^{*}$, we have that $\alpha \cdot f_{g}(x) \neq f_{g}(y)$ for all $x \in\left(2^{\omega}\right)^{\Gamma_{j}}$ extending $r$ and $y \in\left(2^{\omega}\right)^{\Gamma_{j}}$ extending $s$.

We can find such a $p^{*}$ in the following way. Let $\sigma$ be a finite initial segment of $\Gamma_{j} \times \omega$ (according to our fixed enumeration) that contains the three sets $\left\{\left(\beta^{-1} \gamma, n\right):(\gamma, n) \in \operatorname{dom}(r) \wedge \beta \in S_{t(\alpha)}\right\}, \operatorname{dom}(s)$, and $\bigcup\{\operatorname{dom}(t)$ : $t \in \operatorname{dom}(p)\}$. Let $k$ be sufficiently large so that

$$
\min \left(N_{\alpha, j, k}\right)>\sup (\{|p(t)|: t \in \operatorname{dom}(t)\}) \text { and }|\operatorname{dom}(p)|^{S_{k}}<2^{\left|N_{\alpha, j, k}\right|}
$$

Let $l=\max \left(N_{1, j, k}\right)$. Let $q$ be an extension of $p$ such that

$$
\operatorname{dom}(q)=\left\{t \in 2^{<\Gamma_{j} \times \omega}: \operatorname{dom}(t) \subseteq \sigma\right\}
$$

and for every $t \in \operatorname{dom}(q)$ with $\operatorname{dom}(t)=\sigma, q(t)$ has length $l$, and consists of a string in $\operatorname{ran}(p)$ followed by finitely many zeroes. Note that if $g:\left(2^{\omega}\right)^{\Gamma_{j}} \rightarrow 2^{\omega}$ is a continuous function extending $q$, then for every $x \in\left(2^{\omega}\right)^{\Gamma_{j}}$, we have that $g(x)\lceil l$ must be an element of $\operatorname{ran}(p)$ followed by finitely many zeroes. Our desired $p^{*}$ will be equal to some $q$ except on extensions of $s$.

Now if $n \in N_{t\left(\alpha^{-1}\right), j, k}$, then the $n$th bit of $\left(\alpha \cdot f_{g}(x)\right)(1)(j)$ will have been defined in the game $G_{j}^{A_{j}}\left(\left(n_{k}\right)\right)$ by player II on turn $k$, and hence will depend only on the values of $g(\beta \cdot x)\left(n^{\prime}\right)$ for $\beta \in \Gamma_{j}$ with $t(\beta) \leq k$, and $n^{\prime}$ such that

1. $\left\langle j, n^{\prime}\right\rangle<n_{k+1}$ if $t(\beta) \leq t(\alpha)$. 
2. $\left\langle j, n^{\prime}\right\rangle<n_{k-t\left(\alpha^{-1}\right)}$ if $t(\beta)>t(\alpha)$.

Suppose $g$ is a generic continuous function extending $q$, and $x$ extends $r$. Then for any $\beta \in \Gamma_{j}$,

1. if $t(\beta) \leq t(\alpha)$, then since $\beta \cdot x$ is incompatible with $x, g(x) \uparrow\left\{n^{\prime}\right.$ : $\left.\left\langle j, n^{\prime}\right\rangle<n_{k+1}\right\}$ must be an element of $\operatorname{dom}(p)$ followed by finitely many 0s.

2. if $t(\beta)>t(\alpha)$, then $g(x) \uparrow\left\{n^{\prime}:\left\langle j, n^{\prime}\right\rangle<n_{k-t\left(\alpha^{-1}\right)}\right\}$ must also be an element of $\operatorname{dom}(p)$ followed by finitely many $0 \mathrm{~s}$.

Hence, there are at most $w=|\operatorname{ran}(p)|^{\left|S_{k}\right|}$ ways to associate an element in $\operatorname{ran}(p)$ to each $\beta \in S_{k}$, and so at most $\left.|\operatorname{ran}(p)|\right|_{k} \mid$ possible values that $\alpha \cdot f_{g}(x)(i)(j)\left\lceil N_{\alpha, j, k}\right.$ could take. Let $v_{0}, \ldots, v_{w-1}: N_{\alpha, j, k} \rightarrow 2$ enumerate these possibilities. Let $p^{*} \subseteq p$ be a condition with $\operatorname{dom}\left(p^{*}\right)=\operatorname{dom}(q)$ and such that for every $t$ with $\operatorname{dom}(t)=\sigma$, if $t$ is incompatible with $s$, then $p^{*}(t)=q(t)$. Otherwise, if $t$ extends $s$, then $p^{*}(t)$ is incompatible with $v_{0}, \ldots, v_{w-1}$.

Theorems 1.1 and 1.2 from the introduction are now easy corollaries of Lemma 4.7. Note that as we discussed at the beginning of the introduction, $\prod_{i} 2^{\omega}$ and $2^{\omega}$ are homeomorphic, so it is fine to use the space $\left(\prod_{i} 2^{\omega}\right)^{*_{i} \Gamma_{i}}$ instead of $\left(2^{\omega}\right)^{*} \Gamma_{i}$.

Proof of Theorem 1.1. Let $\left(n_{k}\right)_{k \in \omega}$ be a good sequence, and $\left\{A_{i}\right\}_{i \in I}$ be a Borel partition of $\left(\prod_{i} 2^{\omega}\right)^{*_{i} \Gamma_{i}}$. By Lemma 2.5 but for the game $G_{i}\left(\left(n_{k}\right)\right)$, there must be some $j \in I$ so that player II wins the game $G_{j}^{A_{j}}\left(\left(n_{k}\right)\right)$. Now apply Lemma 4.7.

The proof of Theorem 1.2 simply adds the ideas from the proof of Lemma 2.12.

Proof of Theorem 1.2. Let $\left(n_{k}\right)_{k \in \omega}$ be a good sequence, and $\left\{A_{i}\right\}_{i \in I}$ be a Borel partition of Free $\left(\left(\prod_{i} 2^{\omega}\right)^{*_{i} \Gamma_{i}}\right)$. Now define the partitions $\left\{B_{i}\right\}_{i \in I}$ and $\left\{C_{i}\right\}_{i \in I}$ identically as in Lemma 2.12, and let $A_{i}^{\prime}=A_{i} \cup B_{i} \cup C_{i}$ so that $\left\{A_{i}^{\prime}\right\}_{i \in I}$ is a Borel partition of $\left(\prod_{i} 2^{\omega}\right)^{*_{i} \Gamma_{i}}$. Player II wins $G_{j}^{A_{j}^{\prime}}\left(\left(n_{k}\right)\right)$ for some $j \in I$, so we can apply Lemma 4.7 to obtain a continuous equivariant injective function $f:\left(2^{\omega}\right)^{\Gamma_{i}} \rightarrow\left(\prod_{i} 2^{\omega}\right)^{*_{i} \Gamma_{i}}$. But then arguing identically as in the proof of Lemma 4.7 $\operatorname{ran}\left(f\left\lceil\operatorname{Free}\left(\left(2^{\omega}\right)^{\Gamma_{i}}\right)\right)\right.$ must be contained in $A_{j}$. 
We will make a remark here on the extent to which the function $g$ in the proof of Lemma 4.7 depends on the winning strategy for player II in the game $G_{j}$.

Remark 4.8. We have remarked in the proof of Lemma 4.7 above that a sufficiently generic function $g:\left(2^{\omega}\right)^{\Gamma_{j}} \rightarrow 2^{\omega}$ will have the property that there exists a function $\rho_{j}: \omega \rightarrow \omega$ such that for all $x \in\left(2^{\omega}\right)^{\Gamma_{j}}$ and $n \in \omega$, $x(1)(n)=g(x)\left(\rho_{j}(n)\right)$. We remark here these functions $\rho_{j}$ can be chosen so that they will work regardless of what the winning strategy for player II is in the game $G_{j}^{A_{j}}\left(\left(n_{k}\right)\right)$.

Precisely, we mean that if we fix a good sequence $\left(n_{k}\right)_{k \in \omega}$, then there exists a choice of function $\rho_{i}: \omega \rightarrow \omega$ for each $i \in I$ such that if player II wins the game $G_{j}^{A_{j}}\left(\left(n_{k}\right)\right)$, then there is a continuous function $g:\left(2^{\omega}\right)^{\Gamma_{j}} \rightarrow 2^{\omega}$ such that $x(1)(n)=g(x)\left(\rho_{j}(n)\right)$ for all $x$ and $n$, and an injective continuous $\Gamma_{j}$-equivariant function $f:\left(2^{\omega}\right)^{\Gamma_{j}} \rightarrow A_{j}$ that is an embedding of $E\left(\Gamma_{j}, 2^{\omega}\right)$ into $E\left(*_{i} \Gamma_{i}, \prod_{i} 2^{\omega}\right)$ and such that $f_{g}(x)(1)(j)=g(x)$ for every $x \in\left(2^{\omega}\right)^{\Gamma_{j}}$.

The reason this is true is that when we extend the condition $p$ to $p^{*}$ in the proof of Lemma 4.7, the condition $p^{*}$ will depend on the winning strategy for player II in the game $G_{j}$ (since $p^{*}$ must diagonalize against how the strategy works), however the length of elements in the range of $p^{*}$ will always be $l$ independent of what the winning strategy is. Hence, if we choose $\rho_{j}$ in advance to be sufficiently fast growing, we will be able to alternate meeting the dense sets defined in the proof of Lemma 4.7 (where we extend $p$ to $\left.p^{*}\right)$ with dense sets ensuring that $x(1)(n)=g(x)\left(\rho_{j}(n)\right)$.

Next, we will show how we can combine Lemma 4.7 and Lemma 2.7 to construct a Borel cardinality preserving ultrafilter for $E_{\infty}$, which will give a new way of proving many of the theorems from Section 3 of [30].

Lemma 4.9. Suppose $I=2$ and $\Gamma_{0}$ and $\Gamma_{1}$ are countably infinite groups, and $\left(n_{k}\right)_{k \in \omega}$ is strictly increasing. Let $\mathcal{S}$ be the $\sigma$-algebra of $E\left(*_{i} \Gamma_{i}, \prod_{i} 2^{\omega}\right)$ invariant Borel sets. Define $U \subseteq \mathcal{S}$ to be the set of $A \in \mathcal{S}$ such that player II has a winning strategy in $G_{0}^{A}\left(\left(n_{k}\right)\right)$. Then $U$ is a $\sigma$-complete ultrafilter.

Proof. First, it is clear that if $A \in U$ and $B \supseteq A$, then $B \in U$. Now if $B_{1}, B_{2}, \ldots \in U$ then we claim $\left(\cap_{m \geq 1} B_{m}\right)^{\mathrm{c}} \notin U$. This is by contradiction, if $\left(\cap_{m \geq 1} B_{m}\right) \in U$, then by Lemma 2.7. we could obtain a $y$ that is an outcome of a winning strategy for player II in $G_{0}^{\left(\cap_{m \geq 1} B_{m}\right)}\left(\left(n_{k}\right)\right)$ and so that $\gamma_{0, i} \cdot y$ is an outcome of a winning strategy for player II in $G_{0}^{B_{m}}\left(\left(n_{k}\right)\right)$, for each $m \geq 1$. Hence, $y \in \cap_{k \geq 1} B_{k}$, but $\gamma_{0, k} \cdot y \in B_{k}$ for all $k \geq 1$, which is a contradiction, since the $B_{k}$ are invariant. 
An analogous argument shows that if $B_{1}, B_{2}, \ldots \notin U$ then, $\left(\cap_{m \geq 1} B_{m}\right)^{\mathrm{c}} \in$ $U$; if player II does not have a winning strategy in $G_{0}^{A}\left(\left(n_{k}\right)\right)$, then player II has a winning strategy in $G_{1}^{A^{\mathrm{c}}}\left(\left(n_{k}\right)\right)$ by Lemma 2.5. Note that here we are using the fact observed above that both Lemmas 2.5 and 2.7 adapt to the general game $G_{i}\left(\left(n_{k}\right)\right)$.

Now if $\Gamma_{0}=\Gamma_{1}=\mathbb{F}_{\omega}$, then Theorem 4.7 implies that the ultrafilter from Lemma 4.9 preserves Borel cardinality. Hence, we have a new proof of the following theorem, by applying Proposition 4.4.

Theorem $4.10([30]) . E_{\infty}$ is $2^{\aleph_{0}}$-indivisible.

In addition, Lemma 4.7 gives us a new way of proving a couple of the other theorems from Section 3 of [30]. For example, we have the following:

Theorem 4.11 ([30, Corollary 3.1]). If $E$ is a universal countable Borel equivalence relation on a standard Borel space $X$, and $\left\{B_{i}\right\}_{i \in \omega}$ is a Borel partition of $X$, then there must exist a $B_{i}$ such that $E\left(\mathbb{F}_{\omega}, 2^{\omega}\right) \sqsubseteq_{B} E \uparrow B_{i}$.

Proof. Let $g$ be a Borel reduction from $E\left(*_{i<\omega} \mathbb{F}_{\omega}, \prod_{i<\omega} 2^{\omega}\right)$ to $E$. By Lusin-Novikov uniformization [22, Theorem 18.10] we can partition each $g^{-1}\left(B_{i}\right)$ into countably many Borel sets on which $g$ is injective. Let $\left\{A_{j}\right\}_{j \in \omega}$ be the union of these countably many sets over every $i \in \omega$. Now by Lemma 4.7, find a $j \in \omega$ and a Borel embedding $f$ of $E\left(\mathbb{F}_{\omega}, 2^{\omega}\right)$ into $E\left(*_{i<\omega} \mathbb{F}_{\omega}, \prod_{i<\omega} 2^{\omega}\right) \uparrow A_{j}$. Then $g \circ f$ is an injective reduction of $E\left(\mathbb{F}_{\omega}, 2^{\omega}\right)$ to $E \uparrow B_{i}$ for some $i$.

This theorem was used in [30] to also obtain the corollary that if $E$ is a universal (under $\leq_{B}$ ) countable Borel equivalence relation, then $F \sqsubseteq_{B} E$ for every countable Borel equivalence relation $F$.

Of course, our proofs above are not so far from those of [30]. In particular, every ultrafilter given by Lemma 4.9 refines Martin's ultrafilter on the Turing degrees in the following way.

Proposition 4.12. Let $\Gamma_{0}, \Gamma_{1}$ be countably infinite computable groups, and $\left(n_{k}\right)_{k \in \omega}$ be a computable good sequence. It is clear that $\left(\prod_{i} 2^{\omega}\right)^{*_{i} \Gamma_{i}}$ is effectively homeomorphic to $2^{\omega}$, and viewed this way, the shift equivalence relation $E\left(*_{i} \Gamma_{i}, \prod_{i} 2^{\omega}\right)$ is a subequivalence relation of Turing equivalence. Now suppose that $A$ is a Borel subset of $\left(\prod_{i} 2^{\omega}\right)^{*_{i} \Gamma_{i}}$ that is Turing invariant, and hence $E\left(*_{i} \Gamma_{i}, \prod_{i} 2^{\omega}\right)$-invariant. Then $A$ is in Martin's ultrafilter if and only if $A$ is in the ultrafilter $U$ given by Lemma 4.9 . 
Proof. Suppose $s$ is a winning strategy for player II in the game $G_{j}^{A}$. By Lemma 2.7. for every $z \in 2^{\omega}$ there exists a $y$ that is an outcome of the strategy $s$ such that $y(1)(j)=z$. Hence $A$ must contain reals of arbitrarily large Turing degree and hence must be in Martin's ultrafilter. Conversely, if $A$ is in Martin's ultrafilter, then it must also be in the ultrafilter $U$ from Lemma 2.7, as $A^{\mathrm{c}} \in U$ would imply $A^{\mathrm{c}}$ is in Martin's ultrafilter.

Indeed, our game in Definition 2.4 is actually Martin's game from [31] if $A$ is Turing invariant. The bits of $y$ are partitioned into two computable sets: one set that player I determines, and one set that player II determines, and then player II wins if and only if $y$ is in $A$.

\section{$4.3 \mathcal{K}$-structurable equivalence relations}

In this section we use the terminology and notation of [17, Section 2.5]. Suppose $L=\left\{R_{i}: i \in I\right\}$ is a countable relational language and $X_{L}$ is the associate space of $L$-structures whose universe is some set $I \leq \omega$. Let $\mathcal{K} \subseteq$ $X_{L}$ be a Borel class of $L$-structures closed under isomorphism. By a theorem of Lopez-Escobar, such $\mathcal{K}$ are exactly those classes of structures defined by some $L_{\omega_{1}, \omega}$ sentence [22, Theorem 16.8]. A countable Borel equivalence relation $E$ on a standard Borel space $X$ is said to be Borel $\mathcal{K}$-structurable if there is a Borel assignment of a $\mathcal{K}$-structure to each $E$-class whose universe is that class. Precisely, we mean that there are Borel relations $\left\{Q_{i}: i \in I\right\}$ on $X$ where $Q_{i}$ and $R_{i}$ have the same arity for all $i \in I$, and so that for each $E$-class $C$, the structure whose universe is $C$ and whose relations are $Q_{i} \uparrow C$ is isomorphic to some structure in $\mathcal{K}$.

Recall that if $E$ and $F$ are Borel equivalence relations on the standard Borel spaces $X$ and $Y$, then a Borel homomorphism $f: X \rightarrow Y$ from $E$ to $F$ is said to be class bijective if for every $E$-class $C, f \uparrow C$ is a bijection between $C$ and some $F$-class. It is easy to see that if there is a class bijective homomorphism Borel homomorphism from $E$ to $F$, and $F$ is Borel $\mathcal{K}$-structurable, then $E$ is also Borel $\mathcal{K}$-structurable.

Many natural classes of countable Borel equivalence relations are exactly the $\mathcal{K}$-structurable equivalence relations for some such class of structures $\mathcal{K}$. For example, the class $\mathcal{K}$ of trees yields the class of treeable equivalence relations. Similarly, the class of hyperfinite equivalence relations corresponds to the class of structures that are increasing sequences of finite equivalence relations whose union is the universe of the structure. Further, given a class $\mathcal{C}$ of $\mathcal{K}$-structurable equivalence relations for some $\mathcal{K}$, the class of equivalence relations that are finite index over elements of $\mathcal{C}$, and the class of equiv- 
alence relations that are increasing unions of elements of $\mathcal{C}$ are also both structurability classes.

Ben Miller has pointed out the following theorem, which generalizes many ad-hoc universality proofs in the field of countable Borel equivalence relations, such as [20, Proposition 7.1] for graphs. The proof we give is a simplified version of our earlier argument suggested by Kechris. Recall that the notation $E \sqsubseteq_{B}^{i} F$ indicates that there is an injective Borel reduction from $E$ to $F$ whose range is $F$-invariant.

Theorem 4.13 (Miller, after Jackson, Kechris, and Louveau [17] and Kechris, Solecki, Todorcevic [20]). If $\mathcal{K}$ is a Borel class of countable relational structures closed under isomorphism, then there is a universal countable Borel $\mathcal{K}$-structurable equivalence relation, which we note $E_{\infty \mathcal{K}}$. That is, given any countable Borel $\mathcal{K}$-structurable equivalence relation $E$, then $E \sqsubseteq_{B}^{i} E_{\infty \mathcal{K}}$.

Proof. Let $E_{\infty}$ be an invariantly universal countable Borel equivalence relation on the space $Y_{\infty}$ so that for all countable Borel equivalence relations $F$, we have that $F \sqsubseteq_{B}^{i} E_{\infty}$ (see [4]). For example, let $E_{\infty}=E\left(\mathbb{F}_{\omega}, 2^{\omega}\right)$. Let $y \mapsto f_{y}$ be a Borel map from $Y_{\infty} \rightarrow\left(Y_{\infty}\right) \leq \omega$ associating to each $y \in Y_{\infty}$ a bijection $f_{y}: I \rightarrow Y_{\infty}$ from some $I \leq \omega$ to the equivalence class $[y]_{\infty}$ of $y$.

Now let $Y_{\infty \mathcal{K}}$ be the set of $(x, y) \in X_{L} \times Y_{\infty}$ such that $x \in \mathcal{K}$, and the universe of $x$ has the same cardinality as $[y]_{E_{\infty}}$. Now $E_{\infty \mathcal{K}}$ is defined to be the equivalence relation on $A$ where $\left(x_{0}, y_{0}\right) E_{\infty \mathcal{K}}\left(x_{1}, y_{1}\right)$ iff $y_{0} E_{\infty} y_{1}$ and the structure on $\left[y_{0}\right]_{\infty}$ induced by pushing forward $x_{0}$ under $f_{y_{0}}$ is equal to the structure on $\left[y_{1}\right]_{\infty}$ obtained by pushing forward $x_{1}$ under $f_{y_{1}}$.

Suppose $F$ is a $\mathcal{K}$-structurable countable Borel equivalence relation on $X$ and let $Q_{0}, Q_{1}, \ldots$ be Borel relations on $X$ giving a $\mathcal{K}$-structuring of $F$. Let $g: X \rightarrow Y_{\infty}$ witness that $F \sqsubseteq_{B}^{i} E_{\infty}$. Then $F \sqsubseteq_{B}^{i} E_{\infty \mathcal{K}}$ via the function $x \mapsto(h(x), g(x))$ where $h: X \rightarrow X_{L}$ is the function sending $x$ to the structure $h(x) \in X_{L}$ on the set $\operatorname{dom}\left(f_{g(x)}\right)$ where

$$
R_{i}\left(n_{0}, \ldots, n_{k}\right) \Longleftrightarrow Q_{i}\left(f_{g(x)}\left(n_{0}\right), \ldots, f_{g(x)}\left(n_{k}\right)\right)
$$

It is also clear that $E_{\infty \mathcal{K}}$ is Borel $\mathcal{K}$-structurable. Define the relations $Q_{i}$ on $A$ witnessing $E_{\infty \mathcal{K}}$ is Borel $\mathcal{K}$-structurable by setting

$$
Q_{i}\left(\left(x_{0}, y_{0}\right), \ldots\left(x_{n}, y_{n}\right)\right) \Longleftrightarrow x_{0} \models R_{i}\left(f_{y_{0}}^{-1}\left(y_{0}\right), f_{y_{0}}^{-1}\left(y_{1}\right), \ldots, f_{y_{0}}^{-1}\left(y_{n}\right)\right)
$$

We have the following trivial corollary of Sacks's theorem that Turing cones have measure zero, which generalizes [30, Theorem 3.10]. 
Theorem 4.14. Suppose $\mathcal{K}$ is a Borel class of countable structures closed under isomorphism, and let $E_{\infty \mathcal{K}}$ be the universal $\mathcal{K}$-structurable equivalence relation on the space $Y_{\infty \mathcal{K}}$. Then if $\mu$ is a Borel probability measure on $Y_{\infty \mathcal{K}}$, there is a $\mu$-null Borel set $A$ so that $E_{\infty \mathcal{K}} \sqsubseteq_{B}^{i} E_{\infty \mathcal{K}} \uparrow A$.

Proof. Our definition of $E_{\infty \mathcal{K}}$ in Theorem 4.13 has $Y_{\infty}=\left(2^{\omega}\right)^{\mathbb{F}_{\omega}}$ which is computably homeomorphic to $2^{\omega}$, and $X_{L}$ also computably homeomorphic to $2^{\omega}$ relative to the language $L$. Hence, it makes sense to discuss computability of elements of the set $Y_{\infty K} \subseteq X_{L} \times Y_{\infty}$ on which $E_{\infty \mathcal{K}}$ is defined.

By the relativized version of Sacks's theorem [34], if $x \in 2^{\omega}$ can compute a representation of $\mu$, and $y>_{T} x$, then the cone $\left\{z \in 2^{\omega}: z \geq y\right\}$ has $\mu$-measure 0 . Choose such a $y$, and note that since $E_{\infty \mathcal{K}}$ is induced by a Borel action of $\mathbb{F}_{\omega}$, there is an invariant Borel embedding $E_{\infty K}$ into $E_{\infty}$ via the function $g(x)(\gamma)=y \oplus \gamma^{-1} \cdot x$. Using $g$ to define an invariant Borel embedding of $F=E_{\infty \mathcal{K}}$ into $E_{\infty \mathcal{K}}$ as in the proof of Theorem 4.13, then the range of the resulting function is contained in the cone $\left\{z: z \geq_{T} y\right\}$, which therefore has $\mu$-measure 0 .

We mention here that there is a wealth of open problems related to how model theoretic properties of $\mathcal{K}$ are related to the Borel cardinality of $E_{\infty \mathcal{K}}$. For example, suppose $\mathcal{K}$ is the isomorphism class of a single structure. Can we characterize when $E_{\infty \mathcal{K}}$ is smooth? How about in the special case when the structure is a Fraïssé Limit?5

\subsection{Ultrafilters for $\mathcal{K}$-structurable equivalence relations closed under independent joins}

Our next goal is to use arguments similar to those in Section 4.2 to obtain ultrafilters for a class of universal $\mathcal{K}$-structurable equivalence relations. The key property $\mathcal{K}$ must have to allow our arguments to work is that the class of $\mathcal{K}$-structurable equivalence relations must be closed under independent joins.

Say that the collection of $\mathcal{K}$-structurable countable Borel equivalence relations is closed under binary independent joins if whenever $E$ and $F$ are independent countable Borel $\mathcal{K}$-structurable equivalence relations on a standard Borel space $X$, then their join $E \vee F$ is also $\mathcal{K}$-structurable Borel equivalence relations. Say that the $\mathcal{K}$-structurable countable Borel equivalence relations are closed under countable independent joins if

\footnotetext{
${ }^{5}$ These questions have been resolved by 3
} 
whenever $\left\{E_{i}\right\}_{i \in I}$ are independent countable Borel $\mathcal{K}$-structurable equivalence relations on the same standard Borel space, then their join $\bigvee_{i \in I} E_{i}$ is also a $\mathcal{K}$-structurable Borel equivalence relation.

Trees, contractible simplicial complexes of dimension $\leq n$, and the class of all countable structures are all examples of classes of structures $\mathcal{K}$ so that the $\mathcal{K}$-structurable Borel equivalence relations are closed under countable independent joins. So are increasing unions of these examples. Locally finite trees and Cayley graphs of groups $\Gamma$ for which $\Gamma * \Gamma \cong \Gamma$ are examples of classes of structures $\mathcal{K}$ so that the $\mathcal{K}$-structurable Borel equivalence relations are closed under binary independent joins.

For the specific case of contractible simplicial complexes of dimension $\leq n$, we note that the universal structurable equivalence relation for this class is Borel bireducible with the equivalence relation of isomorphism of contractible simplicial complexes of dimension $\leq n$ by standard arguments. Gaboriau has shown [1] that these equivalence relations form a proper hierarchy under $\leq_{B}$. See also [16].

Theorem 4.15. Suppose $\mathcal{K}$ is a Borel class of countable structures so that the class of $\mathcal{K}$-structurable Borel equivalence relations is closed under binary independent joins. Then there is an ultrafilter on the invariant Borel sets of $E_{\infty \mathcal{K}}$ that preserves Borel cardinality. Hence, $E_{\infty \mathcal{K}}$ is $2^{\aleph_{0}}$-indivisible.

Proof. The proof of this theorem uses ideas from the proofs of Lemma 2.12, Remark 4.8, and Lemma 4.9.

Let $I=2$, and $\Gamma_{i}=\mathbb{F}_{\omega}$ for $i \in I$. Let $\hat{X}_{i} \subseteq\left(2^{\omega}\right)^{\Gamma_{i}}$ be the range of a class bijective Borel embedding of $E_{\infty} \mathcal{K}$ into $E\left(\Gamma_{i}, 2^{\omega}\right)$, so $E\left(\Gamma_{i}, 2^{\omega}\right) \uparrow \hat{X}_{i}$ is Borel isomorphic to $E_{\infty \mathcal{K}}$ for every $i$.

Fix functions $\rho_{i}: \omega \rightarrow \omega$ as in Remark 4.8, and let $\pi_{i}^{\prime}:\left(\prod_{i} 2^{\omega}\right)^{*_{i} \Gamma_{i}} \rightarrow$ $\left(2^{\omega}\right)^{\Gamma_{i}}$ be the function

$$
\pi_{i}^{\prime}(x)(\gamma)(n)=x(\gamma)(i)\left(\rho_{i}(n)\right)
$$

so that $\pi_{i}^{\prime}$ is $\Gamma_{i}$-equivariant. Note that $\pi_{i}^{\prime}$ is essentially just the function $\pi_{i}$ from Definition 2.6 modified by $\rho_{i}$.

In our proof we will be considering functions $f_{g}:\left(2^{\omega}\right)^{\Gamma_{i}} \rightarrow\left(\prod_{i} 2^{\omega}\right)^{*_{i} \Gamma_{i}}$ as defined in the proof of Lemma 2.12 and Remark 4.8, and so together, these functions will have the property that

$$
\pi_{i}^{\prime}\left(f_{g}(x)\right)=x
$$

Let $X_{i}=\left\{y \in\left(\prod_{i} 2^{\omega}\right)^{*_{i} \Gamma_{i}}: \pi_{i}^{\prime}(y) \in \hat{X}_{i} \wedge \forall \gamma \in \Gamma_{i}\left(\gamma \cdot y \neq y \Rightarrow \gamma \cdot \pi_{i}^{\prime}(y) \neq\right.\right.$ $\left.\left.\pi_{i}^{\prime}(y)\right)\right\}$ so $X_{i}$ is $\Gamma_{i}$-invariant. 
Let $Y$ be the largest invariant set of $y \in Y$ such that $y \in X_{i}$ for every $i \in I$, and let $\left\{C_{i}\right\}_{i \in I}$ be a Borel partition of the complement of $Y$ as in Lemma 2.11. Let $E_{i}$ be the equivalence relation on $Y$ where $x E_{i} y$ if $\exists \gamma \in \Gamma_{i}(\gamma \cdot x=y)$. Let $\hat{Y}$ be the largest $*_{i} \Gamma_{i}$-invariant subset of $Y$ on which the $E_{i}$ are independent. Hence the $E_{i}$ are everywhere non-independent on $Y \backslash \hat{Y}$, so let $\left\{B_{i}\right\}_{i \in I}$ be a Borel partition of $Y \backslash \hat{Y}$ as in Lemma 2.10.

Now for every $i \in I, E_{i} \uparrow \hat{Y}$ is $\mathcal{K}$-structurable, since $\pi_{i}^{\prime}$ gives a class bijective homomorphism from $E_{i} \uparrow \hat{Y}$ to $E\left(\mathbb{F}_{\omega}, \mathbb{F}_{\omega}\right) \uparrow X$ which is $\mathcal{K}_{\text {-structurable. }}$ Hence the join $\bigvee_{i}\left(E_{i} \uparrow \hat{Y}\right)$ on $\hat{Y}$ is also $\mathcal{K}$-structurable by assumption. Let $E=\bigvee_{i}\left(E_{i} \uparrow \hat{Y}\right)$ be the equivalence relation on $\hat{Y}$ generated by the shift action of $*_{i} \Gamma_{i}$.

Given any Borel partition $\left\{A_{i}\right\}_{i \in I}$ of $\hat{Y}$, let $A_{i}=A_{i} \cup B_{i} \cup C_{i}$, so $\left\{A_{i}^{\prime}\right\}_{i \in I}$ will be a Borel partition of the whole space $\left(\prod_{i} 2^{\omega}\right)^{*_{i} \Gamma_{i}}$.

Let $\left(n_{k}\right)_{k \in \omega}$ be a good sequence. Suppose player II wins the game $G_{j}^{A_{j}^{\prime}}\left(\left(n_{k}\right)\right)$ and $f_{g}$ is the associated injective continuous $\Gamma_{j}$-equivariant function $f_{g}:\left(2^{\omega}\right)^{\Gamma_{j}} \rightarrow A_{j}^{\prime}$ constructed by Lemma4.7 and Remark4.8. Then since $\pi_{j}^{\prime}\left(f_{g}(x)\right)=x$ for all $x$, we see that $\operatorname{ran}\left(f_{g} \uparrow \hat{X}_{j}\right)$ is contained in $X_{j}$, and so $\operatorname{ran}\left(f_{g} \uparrow \hat{X}_{j}\right)$ is contained in $\hat{Y}$, since it cannot meet $B_{j}$ by Lemma 2.10 or $C_{j}$ by Lemma 2.11 (as in the proof of Lemma 2.12).

Since $E\left(\Gamma_{i}, 2^{\omega}\right) \uparrow \hat{X}_{j}$ is Borel isomorphic to $E_{\infty \mathcal{K}}$, we have just shown that $E_{\infty \mathcal{K}} \leq_{B} E$, and since $E$ is itself $\mathcal{K}$-structurable and so $E \leq_{B} E_{\infty \mathcal{K}}$, we have that $E_{\infty \mathcal{K}} \sim_{B} E$.

Now the collection of $E$-invariant Borel sets $A$ such that player II wins $G_{0}^{A \cup B_{0} \cup C_{0}}\left(\left(n_{k}\right)\right)$ is an ultrafilter on the $E$-invariant Borel sets by an identical proof to Lemma 4.9. Furthermore, if player II wins this game, then $E_{\infty \mathcal{K}} \leq_{B}$ $E \uparrow A$ by our argument above.

This constitutes part (2) of Theorem 1.10 from the introduction. We finish by proving part (3) of this theorem.

Theorem 4.16. Suppose $\mathcal{K}$ is a Borel class of countable structures closed under isomorphism, so that the class of $\mathcal{K}$-structurable countable Borel equivalence relations is closed under countable independent joins. Let $E_{\infty \mathcal{K}}$ be the universal $\mathcal{K}$-structurable Borel equivalence relation on $Y_{\infty} \mathcal{K}$. Then if $\left\{A_{i}\right\}_{i \in \omega}$ is a Borel partition of $Y_{\infty \mathcal{K}}$ into countably many Borel pieces, there is some $A_{i}$ such that $E_{\infty \mathcal{K}} \sqsubseteq_{B} E_{\infty \mathcal{K}} \uparrow A_{i}$. Hence, for all countable Borel equivalence relations $F, E_{\infty \mathcal{K}} \leq_{B} F$ implies $E_{\infty \mathcal{K}} \sqsubseteq_{B} F$.

Proof. If we let $I=\omega$, then the proof of Theorem 4.15 shows that $E=$ $\bigvee\left(E_{i} \uparrow \hat{Y}\right)$ is $\mathcal{K}$-structurable, and hence $E \sqsubseteq_{B} E_{\infty \mathcal{K}}$, and if $\left\{A_{i}\right\}_{i \in \omega}$ is any 
Borel partition of $\hat{Y}$, then there is some $j$ such that $E_{\infty \mathcal{K}} \sqsubseteq_{B} E \uparrow A_{j}$ (since there must be some $j$ so that player II wins the game $\left.G_{j}^{A_{j} \cup B_{j} \cup C_{j}}\right)$. Hence, since $E$ and $E_{\infty \mathcal{K}}$ are bi-embeddable, the first half of the theorem follows for $E_{\infty} \mathcal{K}$.

Suppose now that $F$ is a countable Borel equivalence relation on the standard Borel space $X$, and $E_{\infty \mathcal{K}} \leq_{B} F$ via the Borel reduction $g: Y_{\infty \mathcal{K}} \rightarrow$ $X$. By Lusin-Novikov uniformization, we can partition $Y_{\infty \mathcal{K}}$ into countably many Borel sets $\left\{A_{i}\right\}_{i<\omega}$ on which $g$ is injective. But then $E_{\infty \mathcal{K}} \sqsubseteq_{B} E_{\infty K} \uparrow$ $A_{i}$ for some $j$.

\section{References}

[1] Scot Adams and Alexander S. Kechris, Linear algebraic groups and countable Borel equivalence relations, J. Amer. Math. Soc. 13 (2000), no. 4, 909-943 (electronic). MR1775739 (2001g:03086)

[2] Alessandro Andretta, Riccardo Camerlo, and Greg Hjorth, Conjugacy equivalence relation on subgroups, Fund. Math. 167 (2001), no. 3, 189-212. MR1815088 (2001m:03091)

[3] Ruiyuan Chen and Alexander Kechris, Structurable equivalence relations, 2016. Preprint.

[4] R. Dougherty, S. Jackson, and A. S. Kechris, The structure of hyperfinite Borel equivalence relations, Trans. Amer. Math. Soc. 341 (1994), no. 1, 193-225. MR1149121 (94c:03066)

[5] Randall Dougherty and Alexander S. Kechris, The universality of recursive isomorphism on $\omega^{\omega}, 1991$. Preprint.

[6] _ How many Turing degrees are there?, Computability theory and its applications (Boulder, CO, 1999), 2000, pp. 83-94. MR1770736 (2001d:03104)

[7] H. A. Dye, On groups of measure preserving transformation. I, Amer. J. Math. 81 (1959), 119-159. MR0131516 (24 \#A1366)

[8] _ On groups of measure preserving transformations. II, Amer. J. Math. 85 (1963), 551-576. MR0158048 (28 \#1275)

[9] Jacob Feldman and Calvin C. Moore, Ergodic equivalence relations, cohomology, and von Neumann algebras. I, Trans. Amer. Math. Soc. 234 (1977), no. 2, 289-324. MR0578656 (58 \#28261a)

[10] Harvey M. Friedman, Higher set theory and mathematical practice, Ann. Math. Logic 2 (1970/1971), no. 3, 325-357. MR0284327 (44 \#1556)

[11] Damien Gaboriau, Invariants $l^{2}$ de relations d'équivalence et de groupes, Publ. Math. Inst. Hautes Études Sci. 95 (2002), 93-150. MR1953191 (2004b:22009)

[12] Sherwood J. Hachtman, Calibrating determinacy strength in levels of the borel hierarchy, 2016. Preprint.

[13] L. A. Harrington, A. S. Kechris, and A. Louveau, A Glimm-Effros dichotomy for Borel equivalence relations, J. Amer. Math. Soc. 3 (1990), no. 4, 903-928. MR1057041 (91h:28023) 
[14] Leo Harrington, Analytic determinacy and $0^{\sharp}$, J. Symbolic Logic 43 (1978), no. 4, 685-693. MR518675 (80b:03065)

[15] G. Hjorth and A. S. Kechris, The complexity of the classification of Riemann surfaces and complex manifolds, Illinois J. Math. 44 (2000), no. 1, 104-137. MR1731384 (2000m:03115)

[16] Greg Hjorth and Alexander S. Kechris, Rigidity theorems for actions of product groups and countable Borel equivalence relations, Mem. Amer. Math. Soc. 177 (2005), no. 833, viii+109. MR2155451 (2006f:03078)

[17] S. Jackson, A. S. Kechris, and A. Louveau, Countable Borel equivalence relations, J. Math. Log. 2 (2002), no. 1, 1-80. MR1900547 (2003f:03066)

[18] Akihiro Kanamori, The higher infinite, Perspectives in Mathematical Logic, SpringerVerlag, Berlin, 1994. Large cardinals in set theory from their beginnings. MR1321144 (96k:03125)

[19] Vladimir Kanovei, Marcin Sabok, and Jindřich Zapletal, Canonical Ramsey theory on Polish spaces, Cambridge Tracts in Mathematics, vol. 202, Cambridge University Press, 2013.

[20] A. S. Kechris, S. Solecki, and S. Todorcevic, Borel chromatic numbers, Adv. Math. 141 (1999), no. 1, 1-44. MR1667145 (2000e:03132)

[21] Alexander S. Kechris, The structure of Borel equivalence relations in Polish spaces, Set theory of the continuum (Berkeley, CA, 1989), 1992, pp. 89-102. MR1233813 (94h:03093)

[22] _ Classical descriptive set theory, Graduate Texts in Mathematics, vol. 156, Springer-Verlag, New York, 1995. MR1321597 (96e:03057)

[23] _ New directions in descriptive set theory, Bull. Symbolic Logic 5 (1999), no. 2, 161-174. MR1791302 (2001h:03090)

[24] Alexander S. Kechris and Benjamin D. Miller, Topics in orbit equivalence, Lecture Notes in Mathematics, vol. 1852, Springer-Verlag, Berlin, 2004. MR2095154 (2005f:37010)

[25] Wolfgang Krieger, On non-singular transformations of a measure space. I, II, Z. Wahrscheinlichkeitstheorie und Verw. Gebiete 11 (1969), 83-97; ibid. 11 (1969), 98119. MR0240279 (39 \#1628)

[26] Andrew S. Marks, Recursion theory and countable Borel equivalence relations, Ph.D. Thesis, 2012.

[27] - Structure in complete sections of the shift action of a residually finite group., 2013. Unpublished note available at http://math.ucla.edu/ marks/

[28] _ A determinacy approach to Borel combinatorics, J. Amer. Math. Soc. 29 (2016), no. 2, 579-600. MR3454384

[29] _ The universality of polynomial time Turing equivalence, 2016. Preprint.

[30] Andrew S. Marks, Theodore A. Slaman, and John R. Steel, Martin's conjecture, arithmetic equivalence, and countable borel equivalence relations, Ordinal definability and recursion theory. The Cabal Seminar Vol. III, 2016, pp. 493-520.

[31] Donald A. Martin, The axiom of determinateness and reduction principles in the analytical hierarchy, Bull. Amer. Math. Soc. 74 (1968), 687-689. MR0227022 (37 \#2607) 
[32] _ Measurable cardinals and analytic games, Fund. Math. 66 (1969/1970), 287291. MR0258637 (41 \#3283)

[33] _ Determinacy, 2013. Draft manuscript.

[34] Gerald E. Sacks, Degrees of unsolvability, 2nd edition, Princeton University Press, Princeton, N.J., 1966.

[35] Ramez L. Sami, Turing determinacy and the continuum hypothesis, Arch. Math. Logic 28 (1989), no. 3, 149-154. MR1008089 (90i:03058)

[36] Brandon Seward and Robin Tucker-Drob, Borel structurability on the 2-shift of a countable group, 2016. To appear in Annals of Pure and Applied Logic.

[37] Theodore A. Slaman, Aspects of the Turing jump, Logic Colloquium 2000, 2005, pp. 365-382. MR2143887 (2006b:03049)

[38] Theodore A. Slaman and John R. Steel, Definable functions on degrees, Cabal Seminar 81-85, 1988, pp. 37-55. MR960895 (89m:03033)

[39] Simon Thomas, Some applications of superrigidity to Borel equivalence relations, Set theory (Piscataway, NJ, 1999), 2002, pp. 129-134. MR1903855 (2003j:03061)

[40] _ Martin's conjecture and strong ergodicity, Arch. Math. Logic 48 (2009), no. 8, 749-759. MR2563815 (2011g:03120)

[41] Popa superrigidity and countable Borel equivalence relations, Ann. Pure Appl. Logic 158 (2009), no. 3, 175-189. MR2500091 (2009m:03074)

[42] _ Universal Borel actions of countable groups, Groups Geom. Dyn. 6 (2012), no. 2, 389-407. MR2914864

[43] Simon Thomas and Boban Velickovic, On the complexity of the isomorphism relation for finitely generated groups, J. Algebra 217 (1999), no. 1, 352-373. MR1700491 (2000i:20001)

[44] Jay Williams, Universal countable Borel quasi-orders, J. Symb. Log. 79 (2014), no. 3, 928-954. MR3305823

[45] W. Hugh Woodin, The axiom of determinacy, forcing axioms, and the nonstationary ideal, revised, de Gruyter Series in Logic and its Applications, vol. 1, Walter de Gruyter GmbH \& Co. KG, Berlin, 2010. MR2723878 (2011g:03002)

[46] Robert J. Zimmer, Ergodic theory and semisimple groups, Monographs in Mathematics, vol. 81, Birkhäuser Verlag, Basel, 1984. MR776417 (86j:22014) 